\title{
Proinflammatory P2Y14 receptor inhibition protects against ischemic acute kidney injury in mice
}

\author{
Maria Agustina Battistone, ${ }^{1}$ Alexandra C. Mendelsohn, ${ }^{1}$ Raul German Spallanzani, ${ }^{2,3}$ Andrew S. Allegretti, ${ }^{4}$ Rachel N. Liberman, ${ }^{1}$ \\ Juliana Sesma, ${ }^{5}$ Sahir Kalim, ${ }^{4}$ Susan M. Wall, ${ }^{6}$ Joseph V. Bonventre, ${ }^{7}$ Eduardo R. Lazarowski, ${ }^{5}$ Dennis Brown, ${ }^{1}$ and Sylvie Breton ${ }^{1}$ \\ 1Program in Membrane Biology, Division of Nephrology, Department of Medicine, Harvard Medical School and Massachusetts Ceneral Hospital, Boston, Massachusetts, USA. ²Division of Immunology, \\ Department of Microbiology and Immunobiology, Harvard Medical School, Boston, Massachusetts, USA. ${ }^{3}$ Evergrande Center for Immunologic Diseases, Harvard Medical School and Brigham and Women's \\ Hospital, Boston, Massachusetts, USA. 'Division of Nephrology, Department of Medicine, Massachusetts General Hospital, Boston, Massachusetts, USA. ${ }^{5}$ Marsico Lung Institute, Department of Medicine, \\ University of North Carolina, Chapel Hill, North Carolina, USA. ${ }^{6}$ Renal Division, Department of Medicine, Emory University School of Medicine, Atlanta, Ceorgia, USA. ${ }^{7}$ Division of Renal Medicine and ${ }^{8}$ Division \\ of Engineering in Medicine, Harvard Medical School and Brigham and Women's Hospital, Boston, Massachusetts, USA.
}

\begin{abstract}
Ischemic acute kidney injury (AKI), a complication that frequently occurs in hospital settings, is often associated with hemodynamic compromise, sepsis, cardiac surgery, or exposure to nephrotoxins. Here, using a murine renal ischemia/ reperfusion injury (IRI) model, we show that intercalated cells (ICs) rapidly adopted a proinflammatory phenotype after IRI. Wwe demonstrate that during the early phase of AKI either blockade of the proinflammatory P2Y14 receptor located on the apical membrane of ICs or ablation of the gene encoding the P2Y14 receptor in ICs (a) inhibited IRI-induced increase of chemokine expression in ICs, (b) reduced neutrophil and monocyte renal infiltration, (c) reduced the extent of kidney dysfunction, and (d) attenuated proximal tubule damage. These observations indicate that the P2Y14 receptor participates in the very first inflammatory steps associated with ischemic AKI. In addition, we show that the concentration of the P2Y14 receptor ligand UDP-glucose (UDP-CIc) was higher in urine samples from intensive care unit patients who developed AKI compared with patients without AKI. In particular, we observed a strong correlation between UDP-CIc concentration and the development of AKI in cardiac surgery patients. Our study identifies the UDP-GIc/P2Y14 receptor axis as a potential target for the prevention and/or attenuation of ischemic AKI.
\end{abstract}

\section{Introduction}

Acute kidney injury (AKI) is a frequent medical complication seen in hospitalized patients. It is associated with an increased length of hospital stay, the development of chronic kidney disease, and increased risk of mortality (1-4). Over the past decades the rates of AKI have progressively increased worldwide, making it a growing health care burden $(3,5,6)$. Unfortunately, there is no targeted therapy for hospital-acquired AKI other than avoiding potential nephrotoxins and hemodynamic optimization (7-9).

AKI is the consequence of either a direct insult to the kidney or an insult to a distant organ, and multiple primary medical conditions are associated with AKI (10-16). AKI is often associated with renal ischemia, which occurs in the context of multiple organ failure, sepsis, and vascular occlusion (17). Ischemic AKI is particularly common during cardiac surgery requiring cardiopulmonary

Conflict of interest: SB is a cofounder of Kantum Pharma (previously "Kantum Diagnostics Inc."), a company developing a diagnostic and therapeutic combination to prevent and treat acute kidney injury. SB and her spouse own equity in the privately held company. SB and DB are inventors on a patent (US Patent $10,088,489$ ) covering technology that has been licensed to the company through Massachusetts Ceneral Hospital (MCH). SB's and DB's interests were reviewed and are managed by MCH and Partners HealthCare in accordance with their conflict-of-interest policies. Copyright: ( 2020 , American Society for Clinical Investigation.

Submitted: November 5, 2019; Accepted: April 8, 2020; Published: June 8, 2020 Reference information: / Clin Invest. 2020;130(7):3734-3749. https://doi.org/10.1172/JCl134791. bypass $(12,18-21)$. The loss of kidney function is frequently associated with infiltration of circulating innate immune cells into the renal tissue $(14,22-26)$. This results in an inflammatory cascade that enhances and even causes kidney injury, and is rapidly followed by a progressive decline in renal blood flow and glomerular filtration rate, and acute tubular injury (4, 27-29). Renal inflammation is associated with both septic and nonseptic AKI (30), and recent evidence has indicated that activation of innate immunity itself is sufficient to cause AKI (14). In particular, the AKI that follows ischemia/reperfusion injury (IRI) is associated with infiltration of inflammatory cells into the kidney stroma (17, 27, 31). Danger-associated molecular pattern (DAMP) molecules are released following tissue stress or injury and activate pattern recognition receptors, which initiates sterile inflammation (14, 32-34). Activation of pattern recognition receptors by DAMPS induces the production of chemokines, which attract neutrophils and monocytes to the site of inflammation (14). Newly recruited neutrophils and monocytes produce cytotoxic substances such as reactive oxygen species, and they cause microvasculature congestion, which ultimately impairs renal blood flow, creating a sustained ischemic insult (14). This first wave of immune cell infiltration occurs rapidly after injury (27). As such, blockade of this very first step in the inflammatory cascade may offer therapeutic benefits $(7,8,14)$.

DAMPs include, but are not limited to, high-mobility group protein B1 (HMGB1), ATP, DNA, and uridine diphosphate-glucose (UDP-Glc) (34-39). UDP sugars are components of glyco- 


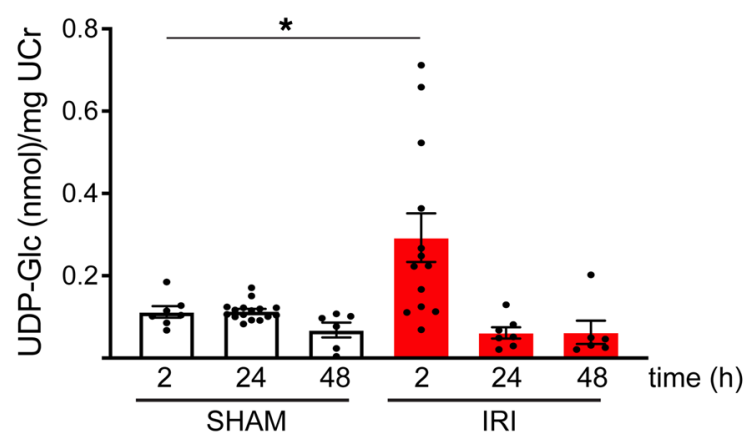

Figure 1. Renal bilateral IRI increases urinary concentration of UDP-GIc. UDPGlc was measured by LC-MS/MS in the urine of mice subjected to bilateral IRI and sham-operated mice. UDP-Glc concentration was normalized for urine creatinine ( $u$ Cr). Each dot represents urine samples pooled from 3 mice. SHAM 2 hours, $n=7$ samples ( 21 mice); SHAM 24 hours, $n=16$ samples (48 mice); SHAM 48 hours, $n=6$ samples ( 18 mice); IRI 2 hours, $n=13$ samples ( 39 mice); IRI 24 hours, $n=7$ samples (21 mice); IRI 48 hours, $n=6$ samples (18 mice). Data are means \pm SEM, analyzed using 2-way ANOVA followed by Tukey's post hoc test. A significant elevation of UDP-Glc/uCr value was detected 2 hours after IRI compared with SHAM (IRI 2 hours vs. SHAM 2 hours, ${ }^{*} P=0.013$ ). No difference was observed 24 hours and 48 hours after IRI. IRI 2 hours vs. IRI 24 hours, $P=0.0006$; IRI 2 hours vs. IRI 48 hours, $P=0.0014$.

sylation reactions, but they can also act as potent agonists of the proinflammatory $\mathrm{P} 2 \mathrm{Y} 14$ receptor when secreted from injured cells $(35,40-44)$. Whereas most nucleotides, such as ATP, are rapidly degraded by ectonucleotidases after their release, extracellular UDP-Glc exhibited high stability in most tissues and cell types investigated $(37,43-45)$. This high stability of UDP-Glc provides a mechanism by which the release of UDP sugars from stressed cells could lead to elevated extracellular concentrations (35), leading to proinflammatory receptor activation. The UDP-Glc-sensing P2Y14 receptor is expressed in several tissues, including the lung and uterus, and epithelial cells can be initiators of inflammation in these organs $(35,41,46)$. Elevated UDPGlc levels and increased numbers of neutrophils were observed in sputum samples from cystic fibrosis patients with moderate or severe lung injury compared with control subjects, and intratracheal instillation of UDP-Glc in mice promoted neutrophil migration into the lung, indicating a link between UDP-Glc and inflammation (43). Similarly, injection of UDP-Glc into the mouse uterus induced a 5 -fold increase in the number of neutrophils present in the endometrium (41). Furthermore, we previously showed that collecting duct intercalated cells (ICs) are sensors of UDP-Glc and mediate the recruitment of neutrophils into the kidney via activation of the P2Y14 receptor located on their apical membrane $(42,47)$.

Here, we used renal IRI as a mouse model of AKI (48) to examine the role of the UDP-Glc/P2Y14 receptor signaling pathway in the pathogenesis of the renal inflammation that leads to kidney injury. Our study shows that blockade of the P2Y14 receptor with a selective inhibitor or ablation of the P2Y14 receptor gene in ICs reduced renal inflammation, attenuated proximal tubule (PT) damage, and improved kidney function following renal IRI. Finally, in a pilot study, we observed that urine UDPGlc levels strongly correlated with the incidence of AKI in inten- sive care unit (ICU) and cardiac surgery patients. This study, therefore, identifies the UDP-Glc/P2Y14 receptor pathway as a potential therapeutic target, which might be used to prevent or alleviate IRI-induced AKI.

\section{Results}

Elevated urine UDP-Glc after renal IRI in mice. Transgenic male mice that express EGFP under the control of the promoter of the IC-specific V-ATPase B1 subunit (ATP6V1B1) gene (B1-EGFP) were subjected to bilateral renal IRI or sham surgery, and they were put in metabolic cages in groups of 3 mice (except for the 2 hours post-IRI group). Urine was collected for the first 2 hours, 24 hours, and 48 hours after operation. UDP-Glc was measured by liquid chromatography-tandem mass spectrometry (LC-MS/MS), and urinary values were normalized for urine creatinine $(\mathrm{uCr})$. We observed a significant increase in the urinary UDP-Glc/uCr ratio in the mice undergoing IRI relative to SHAM during the first 2 hours after IRI. After 24 hours and 48 hours, the urine UDP-Glc/uCr ratios in the IRI groups were similar to the ratios observed in the SHAM groups (Figure 1).

ICs acquire a proinflammatory phenotype after IRI. To determine the response of ICs to experimental IRI, we evaluated their expression of selected proinflammatory transcripts by quantitative PCR (qPCR). $\mathrm{EGFP}^{+}$ICs were isolated by FACS from the kidney medulla of B1-EGFP mice 2 hours, 4 hours, and 24 hours after IRI or sham surgery (Figure 2A). We selected the medulla in order to avoid the isolation of cortical connecting segment cells, which also express EGFP in these mice. We observed upregulation of several chemokines, including $C x c l 1, C x c l 2$, and $C c l 2$, as early as 2 hours after IRI (Figure 2B). Cxcl2 remained elevated up to 24 hours after IRI. The complete gene expression profile of isolated ICs was, therefore, characterized at 2 hours after IRI by RNA sequencing (RNA-Seq). The complete transcriptome data set is shown in Supplemental Table 1 (supplemental material available online with this article; https://doi.org/10.1172/JCI134791DS1). Volcano plots (fold change vs. $P$ value) compare the gene expression profiles of ICs 2 hours after IRI versus SHAM, and show increased expression of several proinflammatory genes, including P2ry14 itself, as well as CxCl1, Cxcl2, Cxcl3, Cxcl5, Cxcl1O, Ccl17, Il1b, Il6, and Il34 (Figure $2 \mathrm{C}$ ). The complete list of genes upregulated after IRI is provided in Supplemental Table 2, where proinflammatory transcripts are indicated in red. A list of selected genes that were downregulated after IRI is provided in Supplemental Table 3. These latter include genes involved in DNA replication, mismatch repair, and metabolism of pyrimidine, glycine, serine, and threonine.

Participation of the P2Y14 receptor in the proinflammatory response of ICs. To determine whether the proinflammatory response of ICs might be related to the increase in urinary UDPGlc after IRI, we next used a potent and highly selective antagonist of the P2Y14 receptor, PPTN (4,7-disubstituted 2-naphthoic acid derivative) $(42,49,50)$, as a means of inhibiting the activation of IC-specific chemokine expression induced by IRI. Because the P2Y14 receptor is located on the apical membrane of ICs (42), and because PPTN and UDP-Glc compete for a common P2Y14 extracellular binding site (35), we first determined whether PPTN is excreted intact into the urine. To make this determination, urine samples were collected following PPTN administration to mice (Figure 3A). PPTN was detected intact in the urine by 

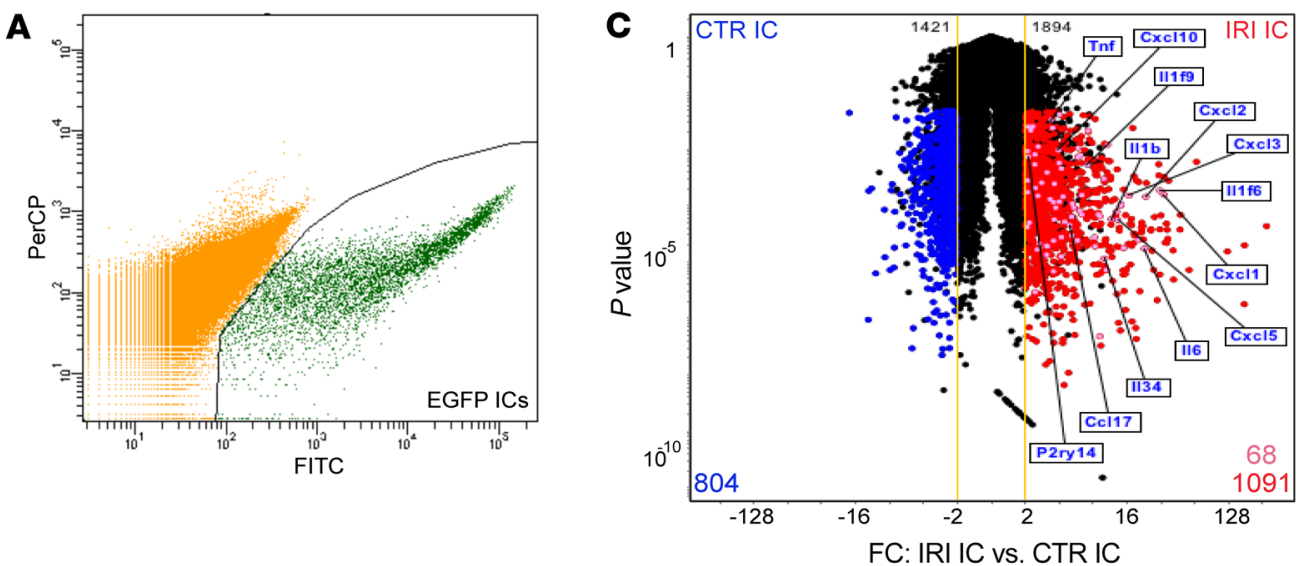

B
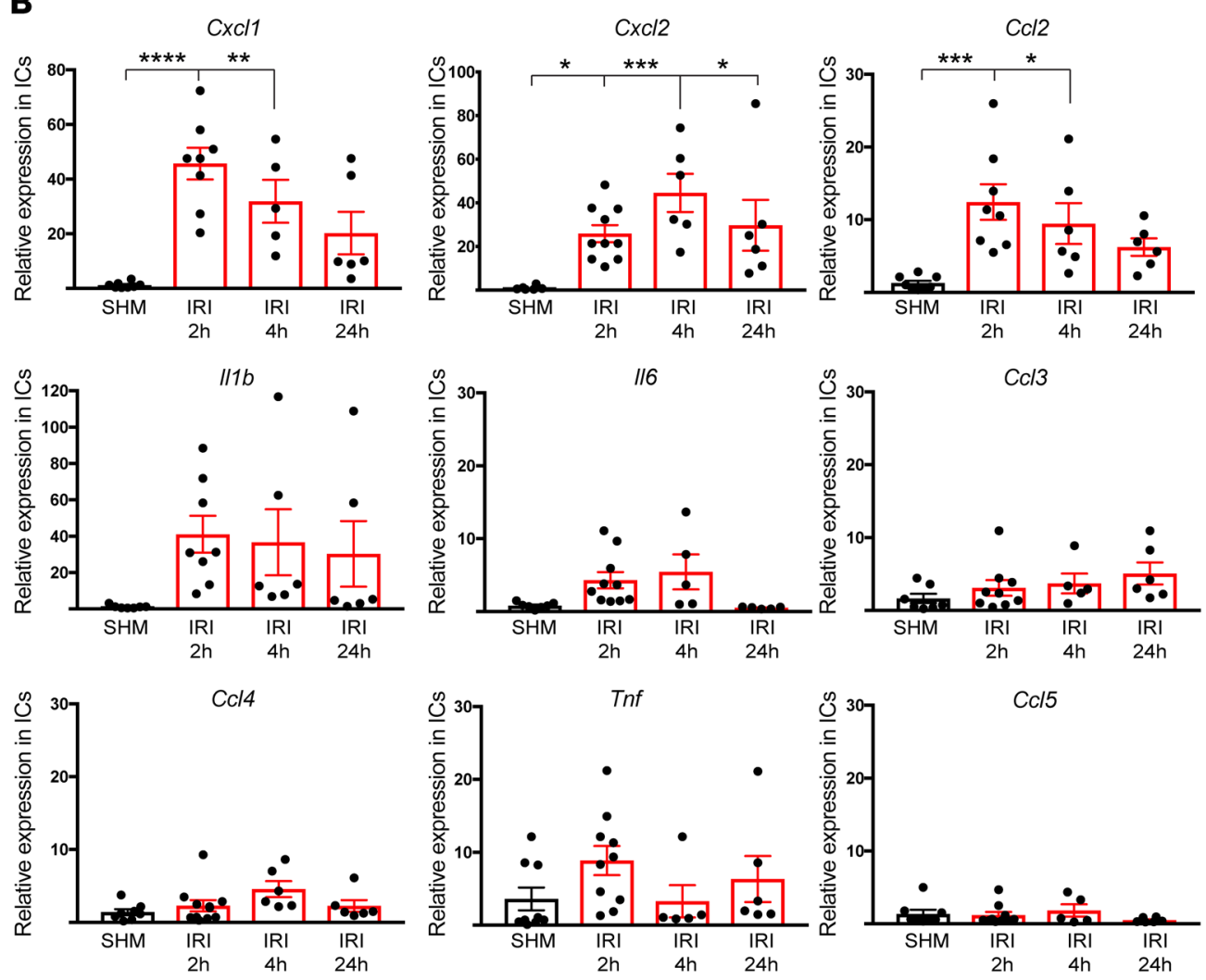

Figure 2. Renal bilateral IRI increases expression of proinflammatory transcripts in ICs. (A) Representative pseudoblot of EGFP+ ICs isolated by FACS from kidney of B1-EGFP mice 2 hours after bilateral IRI. (B) qPCR showing expression of selected proinflammatory chemokines 2,4 , and 24 hours after IRI or sham surgery (SHM). Each dot represents 1 mouse. No difference was detected in SHMoperated mice (2, 4, and 24 hours), and all groups were then combined into a single SHM group. Data are means \pm SEM, analyzed using 1-way ANOVA followed by Dunnett's post hoc test. For $[x c 11$, SHM $(n=8)$, IRI 2 hours ( $n$ $=8)$, IRI 4 hours $(n=5), I R I 24$ hours $(n=6) ;{ }^{* *} P=0.0036,{ }^{* * *} P<0.0001$. For $C x c 12$, SHM $(n=6)$, IRI 2 hours ( $n$ $=10)$, IRI 4 hours $(n=6)$, IRI 24 hours $(n=6) ;{ }^{*} P=0.034,{ }^{* *} P=0.0009$. For CcI2, SHM $(n=8)$, IRI 2 hours $(n=6)$, IRI 4 hours $(n=6)$, IRI 24 hours $(n=$ 6); ${ }^{*} P=0.018,{ }^{* * *} P=0.0006$. For $111 b$, SHM ( $n=7)$, IRI 2 hours $(n=8)$, IRI 4 hours/24 hours $(n=6)$. For $1 / 6$, SHM ( $n$ $=7)$, IRI 2 hours $(n=10)$, IRI 4 hours $/ 24$ hours $(n=5)$. For $C c / 3$, SHM $(n=7)$, IRI 2 hours $(n=9)$, IRI 4 hours $(n=5)$, IRI 24 hours $(n=6)$. For $C \mathrm{C} / 4$, SHM $(n=$ 8), IRI 2 hours ( $n=11)$, IRI 4 hours/24 hours $(n=6)$. For $\operatorname{Tn} f$ and $C \mathrm{c} / 5$, SHM ( $=8)$, IRI 2 hours $(n=10)$, IRI 4 hours ( $n$ $=5)$, IRI 24 hours $(n=6)$. (C) Volcano plots (fold change $[\mathrm{FC}]$ vs. $P$ value) of gene expression profiles of ICS, isolated by FACS 2 hours after IRI (IRI IC) versus SHAM (CTR IC). Each sample of RNA $(n=3)$ was obtained from a pool of 2 kidneys from 2 mice per group. Yellow lines show $\pm 2 \mathrm{FC}$. Genes upregulated after IRI are shown in red, genes downregulated after IRI in blue. Black dots represent transcripts that were not significantly differentially expressed. Data were analyzed using Student's $t$ test, 2 tailed.
LC-MS/MS, and its concentration was $87.8 \pm 24.1 \mathrm{nM} 2$ hours after mice received a single i.v. injection corresponding to the dose of $0.18 \mathrm{mg} / \mathrm{kg}$. A constant higher concentration at around $200 \mathrm{nM}$ was detected at 24 hours and 48 hours, when mice received a daily dose of $4.55 \mathrm{mg} / \mathrm{kg}$ via osmotic minipumps. Control mice received equivalent amounts of the vehicle, DMSO.

To determine whether PPTN could modulate the IC inflammatory response induced by IRI, chemokine expression was assessed by qPCR in ICs isolated by FACS from B1-EGFP mice treated with PPTN or vehicle. ICs were isolated 2 hours after IRI or SHAM procedure. Figure 3B shows that PPTN treatment significantly reduced the increase in IC-specific chemokine expression induced by IRI. No difference in chemokine expression was detected between the SHAM-DMSO and SHAM-PPTN groups, and these 2 groups were, therefore, combined into a single SHAM group in the graph.
Prophylactic inhibition of the $P 2 Y 14$ receptor reduces renal proinflammatory immune cell recruitment after IRI. Renal infiltration of immune cells was quantified by flow cytometry analysis in mice subjected to bilateral IRI. IRI induced significant recruitment of CD 45 $5^{+}$immune cells (Figure 4A), including CD $45^{+} \mathrm{CD} 11 \mathrm{~b}^{+} \mathrm{Ly}_{6 \mathrm{G}}{ }^{+}$ cells with a "neutrophil" phenotype (Figure $4 \mathrm{~B}$ ) and $\mathrm{CD} 45^{+} \mathrm{CD}$ $11 \mathrm{~b}^{+} \mathrm{Ly} 6 \mathrm{C}^{+}$cells with a "monocyte" phenotype (Figure $4 \mathrm{C}$ ), into the kidney tissue as early as 2 hours after surgery. In contrast, PPTN treatment significantly reduced the recruitment of immune cells, neutrophils, and monocytes even at this early time point (Figure $4, \mathrm{~A}-\mathrm{C}$ ). Neutrophil and monocyte recruitment was also lower in the PPTN-treated group compared with the vehicle-treated group 24 hours after IRI (Figure 4, D and E). However, by 48 hours after IRI, neutrophil and monocyte numbers had returned to baseline with values similar in PPTN- and vehicle-treated mice (Figure 4E). 
A

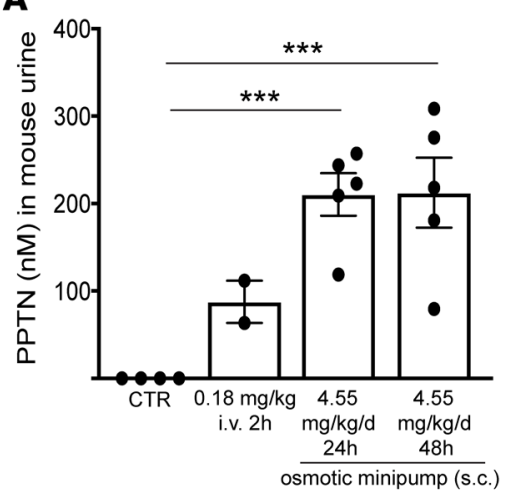

$\mathbf{B}$
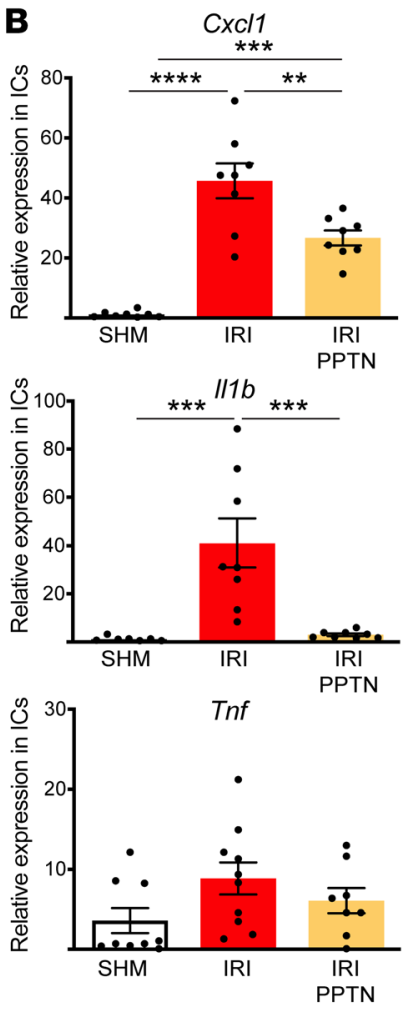
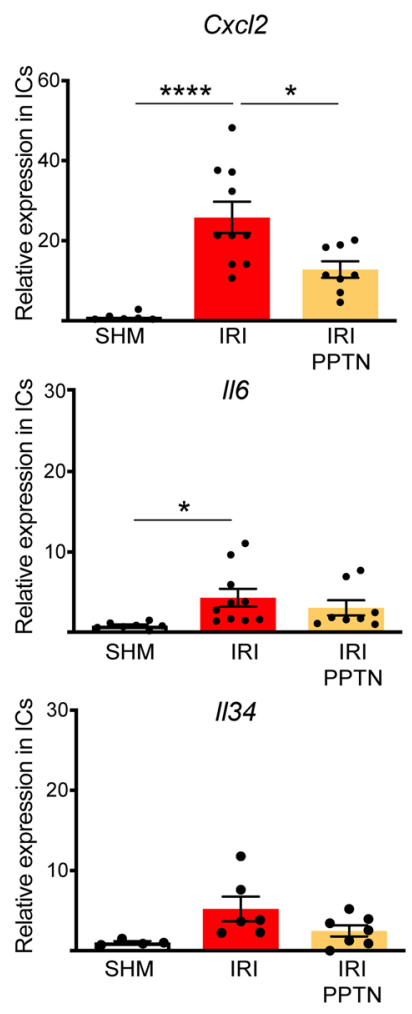
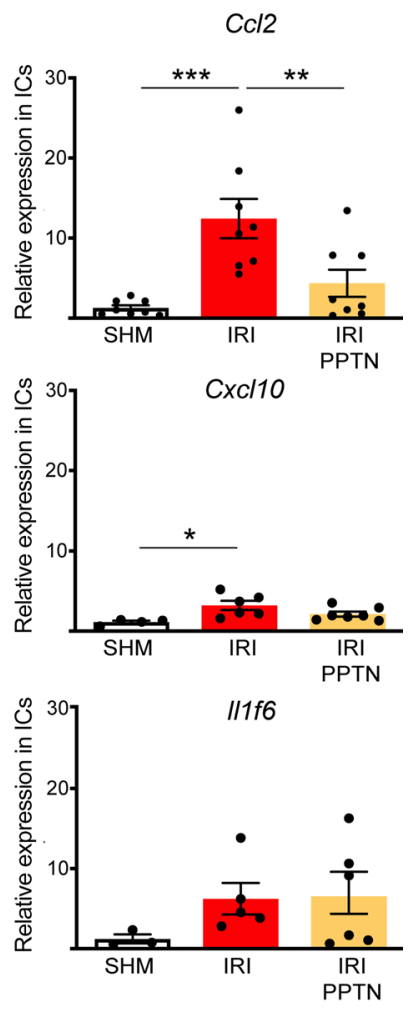

Figure 3. Inhibition of P2Y14 with PPTN attenuates IC-specific upregulation of proinflammatory chemolkines induced by IRI. (A) Concentration of PPTN, a specific P2Y14 antagonist, in the urine of mice treated with a single i.v. injection corresponding to $0.18 \mathrm{mg} / \mathrm{kg}$ (2 hours), or via continuous infusion with ALZET osmotic minipumps implanted s.c. for 24 or 48 hours corresponding to a dose of $4.55 \mathrm{mg} / \mathrm{kg} / \mathrm{d}$, versus controls (CTR; treated with vehicle only). PPTN urinary concentration was measured using LC-MS/MS. Data are means \pm SEM analyzed using 1-way ANOVA and Dunnett's test. CTR vs. $4.55 \mathrm{mg} / \mathrm{kg} / \mathrm{d} 24$ hours ${ }^{* * *} P$ $=0.0007$; CTR vs. $4.55 \mathrm{mg} / \mathrm{kg} / \mathrm{d} 48$ hours ${ }^{* *} P=0.0006$. (B) qPCR analysis of selected chemokines in ICs isolated by FACS 2 hours after IRI versus SHAM. PPTN-treated mice received a dose of $4.55 \mathrm{mg} / \mathrm{kg} / \mathrm{d}$ through osmotic minipumps, while nontreated mice received vehicle. Data are means \pm SEM, analyzed by 1-way ANOVA and Tukey's test. No difference in chemokine expression was detected between the SHAM-vehicle and SHAM-PPTN groups, which were then combined into a single SHAM group (SHM). Some IRI groups are the same as those shown in Figure $2 \mathrm{~B}$ (IRI 2 hours). Each dot represents 1 mouse. For $C x c / 1$, SHM $(n=6)$, IRI $(n=8)$, IRI-PPTN $(n=8) ;{ }^{* *} P=0.0039,{ }^{* *} P=0.0002,{ }^{* * * *} P<0.0001$. For $C x c I 2$, SHM $(n=6)$, IRI $(n=10)$, IRI-PPTN $(n=8) ;{ }^{*} P=0.013,{ }^{* * * *} P<$ 0.0001 . For CcI2, SHM $(n=8)$, IRI $(n=8)$, IRI-PPTN $(n=8) ;{ }^{*} P=0.0092,{ }^{* * *} P=0.0005$. For II1b, SHM $(n=7)$ vs. IRI $(n=8){ }^{* * *} P=0.0006 ;$ IRI vs. IRI-PPTN $(n=$ 8) ${ }^{* * *} P=0.0006$. For $1 / 6$, SHM $(n=7)$, IRI $(n=10)$, IRI-PPTN $(n=8) ;{ }^{*} P=0.042$. For $C x c / 10$, SHM $(n=4)$, IRI $(n=6)$, IRI-PPTN $(n=7) ;{ }^{*} P=0.015$. For Tnf, SHM $(n$ $=9)$, IRI $(n=10)$, IRI-PPTN ( $(n=8)$. For II34, SHM $(n=4)$, IRI $(n=6)$, IRI-PPTN $(n=7)$. For II1f6, SHM $(n=3)$, IRI $(n=5)$, IRI-PPTN $(n=6)$.

Prophylactic inhibition of the P2Y14 receptor reduces the extent of kidney dysfunction after IRI. Further studies explored whether PPTN treatment attenuated the fall in renal function and the rise in markers of kidney injury that follow AKI. Serum creatinine ( $\mathrm{sCr}$ ), blood urea nitrogen (BUN), albuminuria, and the PT injury urine marker KIM-1 were assessed in control mice (SHAM) and mice subjected to renal bilateral IRI. A significant elevation of sCr and BUN was observed following IRI in vehicle-treated mice, as previously shown by several laboratories (refs. 51, 52, and Figure 5, A and B). Mice treated with PPTN showed a significant reduction in $\mathrm{sCr}$ and BUN compared with the SHAM group, indicating preservation of kidney function after IRI. No effect of PPTN was observed at the early 2-hour time point, indicating intrinsic damage caused by ischemia, and sCr did not go up above this initial elevation 24 hours and 48 hours after IRI. IRI also caused albuminuria (Figure 5C) and elevation of KIM-1 urinary concentration (Figure 5D) indicating damage to PTs $(53,54)$, which are known to be among the most affected tubules after IRI. However, PPTN treatment significantly attenuated albuminuria severity and KIM-1 elevation 48 hours after IRI, showing preservation of PT function (Figure 5, C and D).
Prophylactic inhibition of the P2Y14 receptor reduces kidney damage after IRI. Histopathological examination of kidney sections stained using H\&E was conducted to determine the effect of PPTN on kidney tubules following IRI versus SHAM. As shown in Figure 6A, many damaged PTs were detected 24 hours and 48 hours after IRI compared with SHAM in vehicle-treated mice (left). A reduction in the number of damaged PTs was observed in the PPTN-treated groups (right) compared with vehicle-treated groups. This protection was observed 24 hours and 48 hours after IRI. No effect of PPTN was observed in the SHAM group (compare top right and top left). We quantified the number of intact, moderately damaged, and very damaged renal tubules, as shown in Figure 6B. The percentage of intact tubules significantly increased in the PPTN group compared with DMSO at 48 hours after IRI. This was accompanied by a significant reduction in the number of very damaged tubules at 24 hours.

Protection by PPTN following IRI was also demonstrated in kidney sections labeled using phalloidin-Alexa Fluor 647, a marker of F-actin. IRI induced a marked actin depolymerization in the brush border membrane and along the basolateral membrane of 

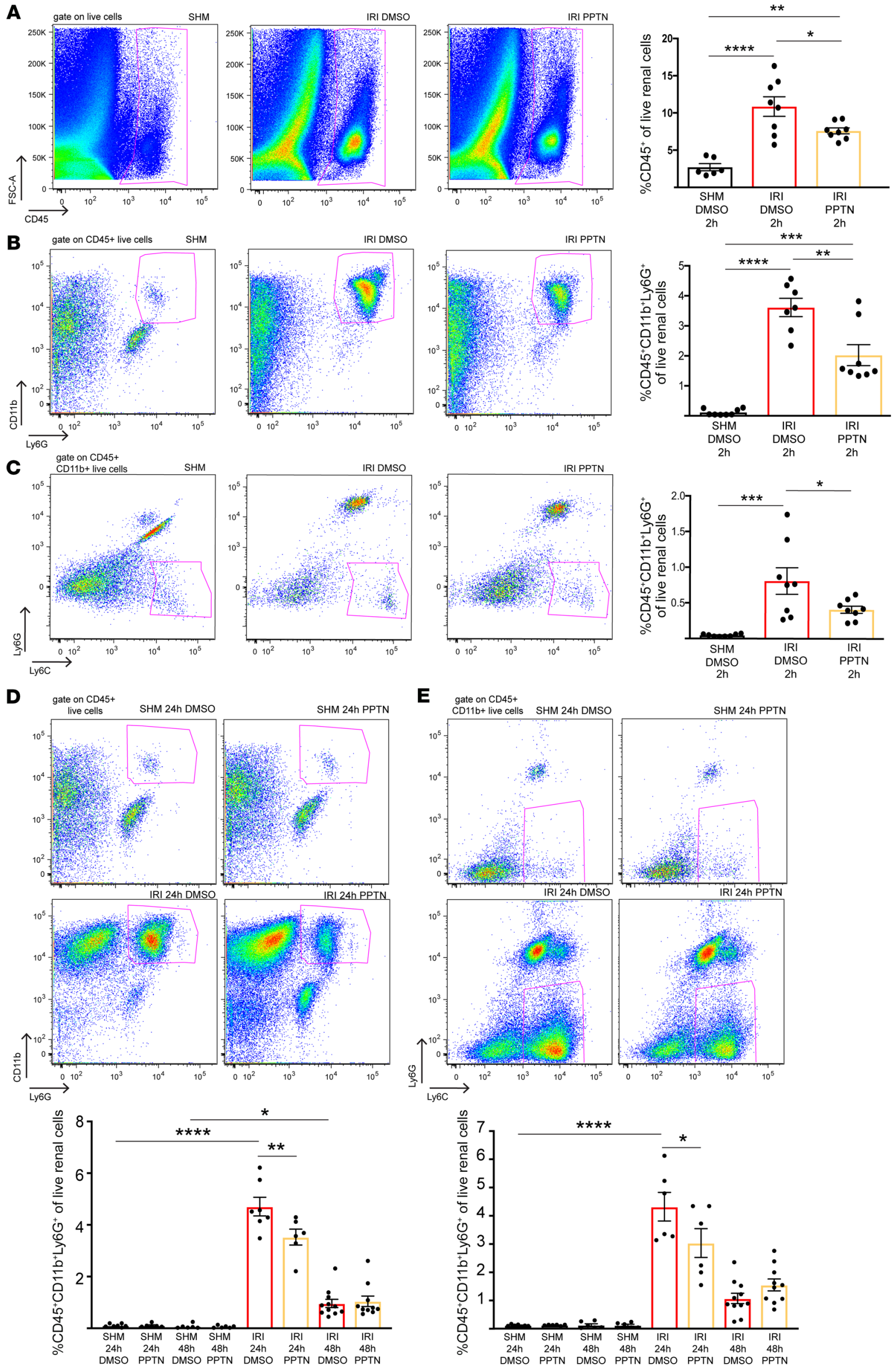
Figure 4. IRI induces the renal recruitment of proinflammatory immune cells, and this process is attenuated by PPTN. (A) Flow cytometry analysis of renal recruitment of live CD45+ immune cells (pink boxes) 2 hours after IRI compared with SHAM. SHM-DMSO $(n=6)$, IRI-DMSO $(n=8)$, IRI-PPTN $(n=8) ;{ }^{*} P=0.037,{ }^{* *} P=0.004,{ }^{* * *} P<0.0001$. (B) Renal recruitment of live neutrophils (CD45+CD11 $\mathrm{b}^{+} \mathrm{Ly} 6 \mathrm{C}^{+}$) relative to the live renal cell population (pink boxes) 2 hours after IRI compared with SHAM. SHM-DMSO $(n=8)$, IRI-DMSO $(n=7)$, IRI-PPTN $(n=8) ;{ }^{* *} P=0.0013,{ }^{* * *} P=0.0001,{ }^{* * *} P<$ 0.0001 . (C) Renal recruitment of live monocytes (CD45 ${ }^{+}$CD11 b ${ }^{+}$Ly6C $^{+}$Ly6C $^{-}$) relative to the live renal cell population (pink boxes) 2 hours after IRI compared with SHAM ( $n=8$ mice in each group). ${ }^{*} P=0.048,{ }^{* *} P=0.0002$.

(D) Renal recruitment of live neutrophils (CD45+CD11 $\mathrm{b}^{+} \mathrm{Ly} 6 \mathrm{C}^{+}$) relative to the renal live cell population 24 hours (left, pink boxes) and 48 hours after IRI compared with SHAM. SHM 24 hours DMSO $(n=7)$, SHM 24 hours PPTN ( $n$ $=7)$, SHM 48 hours DMSO $(n=6)$, SHM 48 hours PPTN $(n=6)$, IRI 24 hours DMSO ( $n=7)$, IRI 24 hours PPTN $(n=6)$, IRI 48 hours DMSO $(n=11)$, IRI 48 hours PPTN $(n=10) ;{ }^{*} P=0.04,{ }^{* *} P=0.0057$, ${ }^{* * *} P<0.0001$. (E) Renal

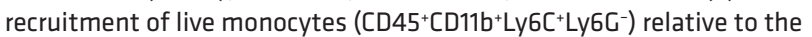
live renal cell population 24 hours (left, pink boxes) and 48 hours after IRI compared with SHAM. SHM 24 hours DMSO $(n=7)$, SHM 24 hours PPTN ( $n$ $=6)$, SHM 48 hours DMSO $(n=6)$, SHM 48 hours PPTN $(n=6)$, IRI 24 hours DMSO $(n=6)$, IRI 24 hours PPTN $(n=6)$, IRI 48 hours DMSO ( $n=11)$, IRI 48 hours PPTN $(n=10) .{ }^{*} P=0.039,{ }^{* * *} P<0.0001$. In all bar graphs, data are means \pm SEM, and each dot represents 1 mouse. (A-C) One-way ANOVA and Tukey's post hoc test. ( $\mathbf{D}$ and $\mathbf{E}$ ) Two-way ANOVA and Tukey's test.

PT cells 24 hours and 48 hours after IRI (Supplemental Figure 1A, left), as previously shown in rats (55). In the treated group, intact brush border membrane and basolateral staining was detected, indicating preservation of the actin cytoskeleton in PT cells (Supplemental Figure 1A, right). Similarly, labeling for the PT-specific scavenger receptor megalin showed loss of apical membrane after IRI (Supplemental Figure 1B, left), and marked protection by PPTN 24 hours and 48 hours after IRI (Supplemental Figure 1B, right). To further assess PT cell polarity, we labeled kidney sections for aquaporin 1 (AQP1), which is located in the brush border and basolateral membrane of intact PT cells (56). IRI induced the redistribution of AQP1 from the plasma membrane into the entire cell body in PTs 24 hours and 48 hours after IRI (Figure 7A, left). This intracellular AQP1 redistribution was less pronounced in the PPTN-treated group (Figure 7A, right). After IRI in both vehicle- and PPTN-treated groups, while some PT cells showed a complete loss of AQP1 polarity with absent apical and basolateral staining after IRI (Figure 7B, very damaged PTs), some cells showed partial redistribution with basolateral staining still visible (Figure 7B, moderately damaged PTs). We quantified the number of intact, moderately damaged, and very damaged PTs based on these AQP1 staining patterns, and found a significant reduction in the number of very damaged PTs together with an increase in the percentage of intact PTs and a decrease in the percentage of very damaged PTs in the treated groups compared with the untreated group 24 hours and 48 hours after IRI.

Deletion of the P2Y14 receptor in ICs confers kidney protection after $I R I$. To confirm the role of the P2Y14 receptor in ICs in renal inflammation and kidney damage leading to ischemic-induced AKI, we next generated an IC-specific P2Y14 receptor-KO mouse by using the Cre-lox system. Transgenic mice expressing Cre under the promoter of the V-ATPase B1 subunit (ATP6V1B1) in ICs (B1 $\left.{ }^{\mathrm{Cre}}\right)(57,58)$ were bred with P2Y14 receptor Lox mice (B6.129-P2ry14<tm1Gac > / Orl; European Mouse Mutant Archive [EMMA], EM:05368). B1 ${ }^{\mathrm{Cre}+}$
P2Y14 ${ }^{\text {flox/+ }}$ mice (IC KO) and B1 ${ }^{\text {Cre- }}$ P2Y14 ${ }^{\text {flox/+ }}$ (IC F/F; controls) were generated. Expression of P2ry14 was assessed by qPCR in ICs that were isolated by FACS based on their expression of the cell surface receptor c-Kit (CD117) (59). A negative selection for CD45 $5^{+}$immune cells, which also express c-Kit, was performed (Figure 8A, left, red dots). A significant reduction in P2ry14 expression was observed in IC KO mice compared with IC F/F mice (Figure 8A, right). IC KO and IC F/F mice were then subjected to bilateral IRI as described above. Deletion of the P2Y14 receptor in ICs improved kidney function, illustrated by a reduction in the IRI-induced $\mathrm{sCr}$ elevation, in comparison with IC F/F mice (Figure 8B), and reduced renal inflammation (Figure 8, C and D) 24 hours after IRI. Protection of renal tubule integrity was shown and quantified in H\&E-stained sections (Figure 8E), and a protective effect of PTs was demonstrated in kidney sections labeled for AQP1 (Figure 8F) or F-actin (Supplemental Figure 2). These results confirm the role of the P2Y14 receptor in ischemic-induced AKI.

Taken together, the results obtained in this preclinical AKI model show the participation of ICs in the rapid onset of renal inflammation, followed by renal dysfunction and PT damage, after an ischemic insult. In our current model (Figure 9), UDP-Glc levels are increased in the urine after renal IRI. Stressed PT epithelial cells, which are the most affected cell type in this model (and potentially other renal cell types), would release UDP-Glc, which then reaches the lumen of the collecting duct, where it activates the P2Y14 receptor located on the apical membrane of ICs. This stimulates the production of proinflammatory chemokines by ICs, followed by rapid renal recruitment of neutrophils and monocytes. Consistent with our flow cytometry analysis, we found many neutrophils positive for N-elastase (Supplemental Figure 3) or Ly6G (Supplemental Figure 4) adjacent to PTs (positive for AQP1) in the DMSO group 24 hours after IRI, and we showed that this neutrophil recruitment was strongly attenuated by PPTN. Very few neutrophils were detected in both SHAM groups (DMSO or PPTN). Many neutrophils were also detected near intact ICs 24 hours after IRI in nontreated mice, while very few neutrophils were detected in PPTN-treated mice (Supplemental Figure 5). These inflammatory cells contribute to creating additional injury by invading PTs, and clogging the microvasculature of the kidney, which perpetuates and aggravates ischemic injury. We observed similar levels of moderate renal tubule damage at 2 hours after IRI in both the DMSO and PPTN groups (Supplemental Figure 6). Altogether, our results show that PPTN confers protection by facilitating repair mechanisms in the renal tubules and/or by attenuating further damage via reduction of postischemia renal inflammation. The latter possibility is supported by our data showing reduced numbers of apoptotic cells 24 hours after IRI in the PPTN versus DMSO groups (Supplemental Figure 7), as well as in the IC KO mice (Supplemental Figure 8). Thus, blocking the P2Y14 receptor with a small molecule or deleting the receptor specifically in ICs significantly reduces the impact of IRI by reducing renal inflammation, preserving kidney function, and reducing kidney tubule damage.

Urine UDP-Glc is elevated in ICU and cardiac surgery patients who develop AKI. Based on these preclinical model data, we next examined whether urinary levels of UDP-Glc in ICU patients could serve as a potential biomarker for AKI. A longitudinal pilot study was conducted using 108 urine samples collected from 35 patients. 
A

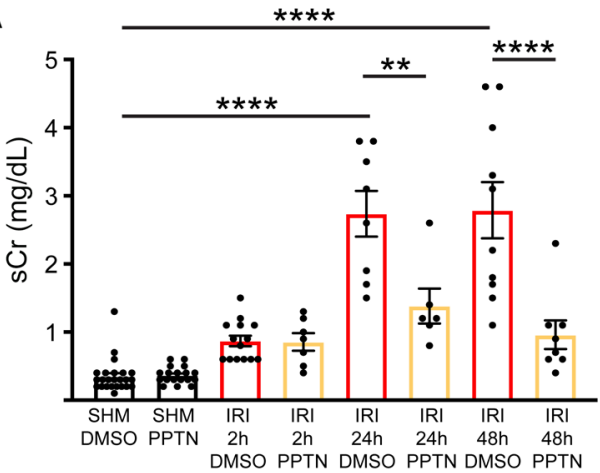

C

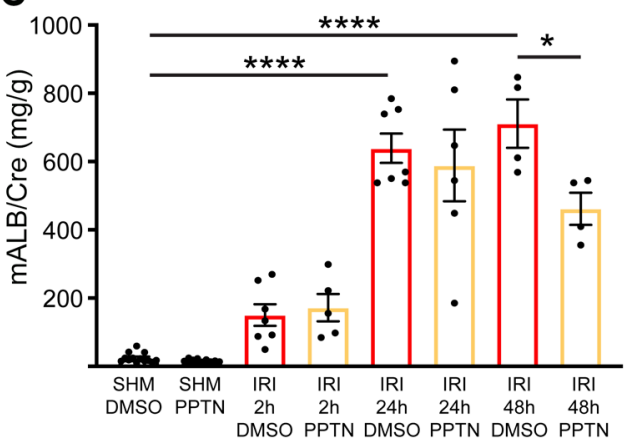

B

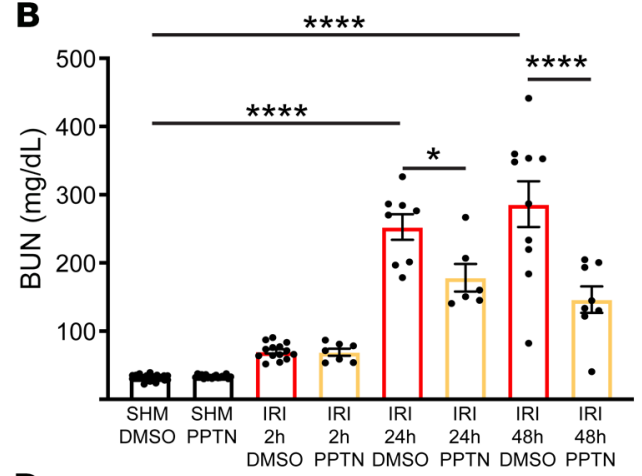

D

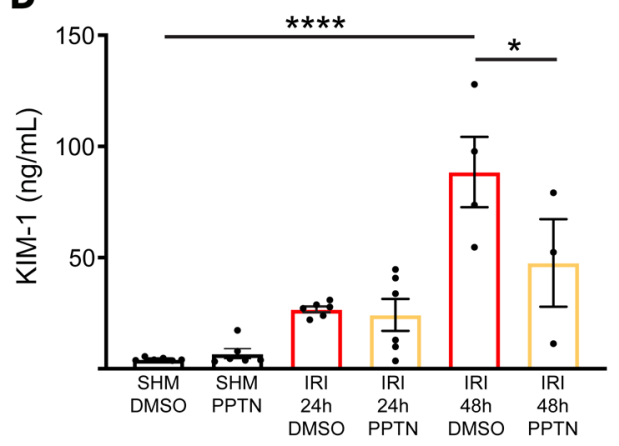

Figure 5. PPTN protects kidney function after IRI. (A) Serum creatinine ( $\mathrm{s} C \mathrm{r}$ ) over time after IRI in vehicle-treated mice (DMSO) versus PPTN. Each dot represents 1 mouse. SHM-DMSO $(n=24)$, SHM-PPTN $(n=18)$, IRI 2 hours DMSO $(n=14)$, IRI 2 hours PPTN $(n=7)$, IRI 24 hours DMSO ( $n=8)$, IRI 24 hours PPTN $(n=6)$, IRI 48 hours DMSO $(n=10)$, IRI 48 hours PPTN $(n=10)$. ${ }^{* *} P=0.0011$, ${ }^{* * *} P<0.0001$. (B) BUN over time after IRI. Each dot represents 1 mouse. SHM-DMSO ( $n=24)$, SHM-PPTN $(n=18)$, IRI 2 hours DMSO $(n=14)$, IRI 2 hours PPTN $(n=7)$, IRI 24 hours DMSO $(n=8)$, IRI 24 hours PPTN $(n=6)$, IRI 48 hours DMSO $(n=10)$, IRI 48 hours PPTN $(n=8) .{ }^{*} P=0.038$, ${ }^{* * *} P<0.0001$. (C) Urine microalbumin/creatinine ratio (mALB/Cre) over time after IRI. Each dot represents urine collection from 3 mice. SHM-DMSO ( $n=13$, representing 39 mice), SHM-PPTN ( $n=9 ; 27$ mice), IRI 2 hours DMSO ( $n=7 ; 21$ mice), IRI 2 hours PPTN ( $n=5 ; 15$ mice), IRI 24 hours DMSO ( $n=7 ; 21$ mice), IRI 24 hours PPTN ( $n=6 ; 18$ mice), IRI 48 hours DMSO ( $n=4 ; 12$ mice), IRI 48 hours PPTN ( $n=4 ; 12$ mice). ${ }^{*} P=0.047,{ }^{* * *} P<0.0001$. (D) Urinary concentration of KIM-1 over time after IRI. Each dot represents urine collection from 3 mice. SHM-DMSO ( $n=6$; 18 mice), SHM-PPTN ( $n=6 ; 18$ mice), IRI 24 hours DMSO ( $n=6 ; 18$ mice), IRI 48 hours PPTN ( $n=3 ; 9$ mice), IRI 48 hours DMSO ( $n=4 ; 12$ mice), IRI 48 hours PPTN $\left(n=3 ; 9\right.$ mice). ${ }^{*} P=0.041,{ }^{* * *} P<0.0001$. For all graphs, data are means \pm SEM, and 2-way ANOVA followed by Tukey's test was performed.

These urine samples were collected daily from patients admitted to the ICU for up to 8 days. Patient characteristics are displayed in Table 1. Briefly, median age was 73 years, $97 \%$ of enrolled patients were white, and $26 \%$ were women. Twenty-six patients underwent elective cardiac surgery requiring cardiopulmonary bypass, and 5 patients had sepsis. Figure 10A displays the peak urinary UDP-Glc concentration observed for each patient stratified by presence or absence of AKI (defined by at least $0.3 \mathrm{mg} / \mathrm{dL}$ increase in $\mathrm{sCr}$ ). In this cohort, 12 patients developed AKI (AKIN stages 1, 2, 3) for an incidence of $34 \%$. When patients progressed to a more advanced AKI during the hospital stay, the higher AKI stage was used. Significantly higher urinary UDP-Glc concentrations were observed in patients who developed AKI versus patients who did not. At a cutoff value of $40 \mathrm{nM}$, UDP-Glc predicted AKI with a sensitivity of $83.3 \%$ and specificity of $78.3 \%$ (positive predictive value of $66.7 \%$ and negative predictive value of $90.0 \%$ ). When cardiac surgery patients were analyzed separately, similar statistic parameters were obtained (Figure 10B), and at a cutoff value of $40 \mathrm{nM}$, UDP-Glc predicted AKI with a sensitivity of $66.7 \%$ and specificity of $90.0 \%$ (positive predictive value of $66.7 \%$ and negative predictive value of $90.0 \%$ ). Results of the receiver operating characteristic (ROC) analysis are shown in Figure 10C for all ICU patients (AUC 0.84; 95\% CI 0.71, 0.97) and Figure 10D for cardiac surgery patients only (AUC $0.91 ; 95 \%$ CI 0.77, 1.00). These results indicate that UDP-Glc is a promising actionable biomarker as a predictor of AKI owing to its high sensitivity and specificity.

\section{Discussion}

Here we report a major role for the UDP-Glc/P2Y14 receptor signaling pathway in driving early renal inflammation, leading to kidney dysfunction and proximal tubule (PT) damage following renal IRI. We show that inhibition of the P2Y14 receptor using a selective antagonist, or deletion of the IC P2Y14 receptor, confers strong kidney protection following bilateral renal IRI. The role of the P2Y14 receptor ligand UDP-Glc as a potential DAMP involved in acute kidney disease is supported by the strong correlation between UDPGlc urinary levels and AKI diagnosis in ICU patients, with a particularly robust statistical association in cardiac surgery patients.

Using transgenic mice that express EGFP driven by the promoter of the IC-specific V-ATPase subunit ATP6V1B1, we characterized the transcriptomic profiles of medullary ICs under control conditions and following renal IRI. Under control conditions, we confirmed expression of several genes previously shown to be expressed in ICs by RNA-Seq of isolated ICs, as well as single-cell RNA-Seq (59). These include high expression levels of the transcription factor Foxi1; the cell surface receptor c-Kit; the cyto- 
A

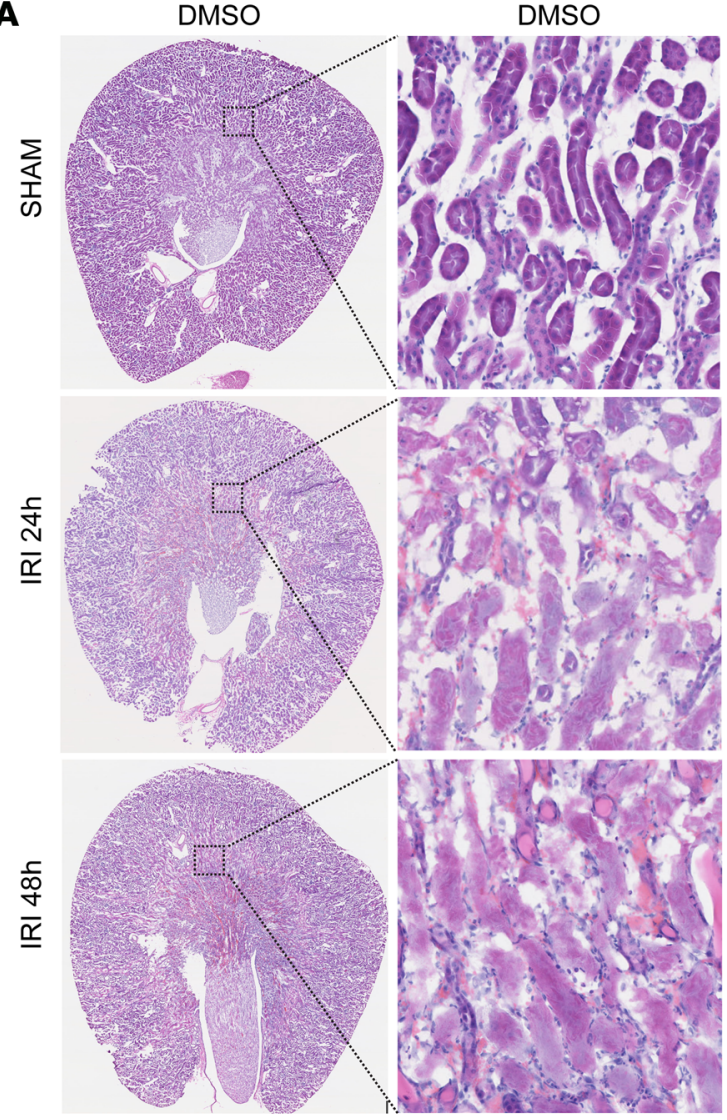

B

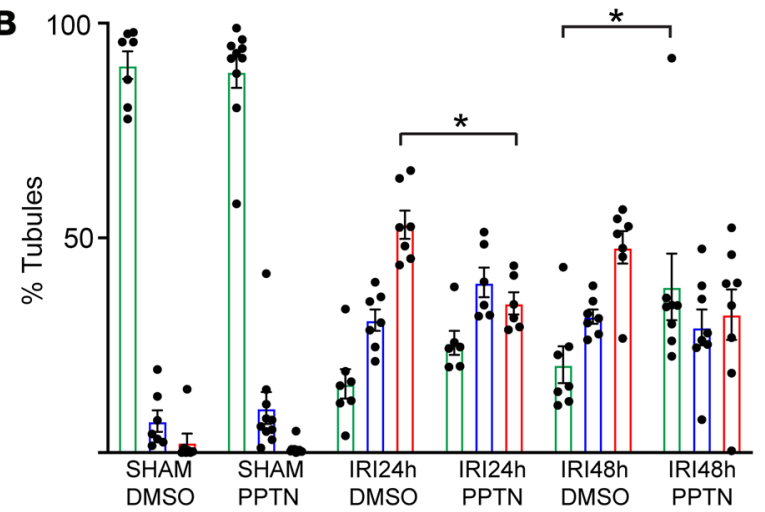

PPTN

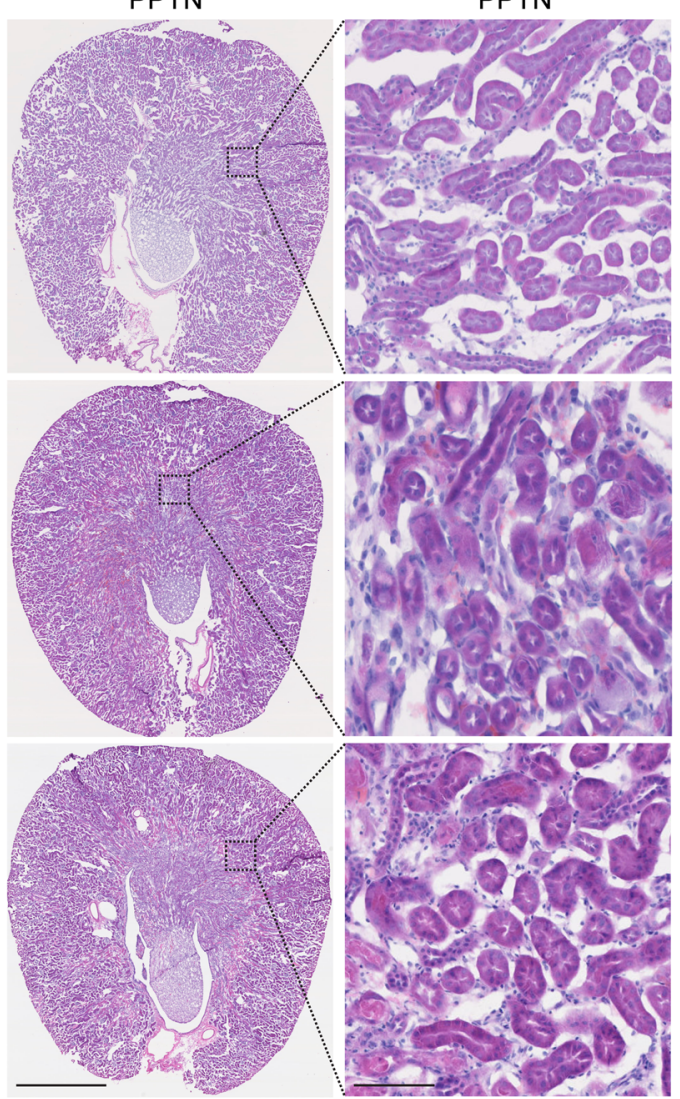

$\square$ Intact tubules

$\square$ Moderately damaged tubules

$\square$ Very damaged tubules

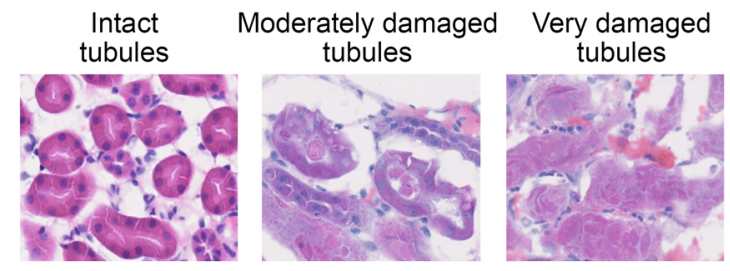

Figure 6. PPTN protects kidney structure and renal tubules after IRI. (A) Kidney sections stained using H\&E in SHAM 24 and 48 hours after IRI. Right panels show higher magnification of the regions delineated by the boxes in left panels. Severe alteration of renal tubule morphology is observed 24 and 48 hours after IRI in the DMSO group. Protection of kidney tubules is observed in the PPTN group versus DMSO at both time points after IRI. No effect of PPTN alone was observed in the SHAM group. Scale bar: $1 \mathrm{~mm}$; inset scale bar: $100 \mu \mathrm{m}$. (B) Quantification of the percentage of intact tubules (green bars) moderately damaged tubules with detectable cellular structures (blue bars), and very damaged tubules with a complete loss of cell architecture (red bars) SHAM-DMSO ( $n=7$ mice), SHAM-PPTN $(n=10)$, IRI 24 hours DMSO $(n=7)$, IRI 24 hours PPTN $(n=6)$, IRI 48 hours DMSO $(n=7)$, IRI 48 hours PPTN $(n=8)$. IRI 24 hours DMSO vs. IRI 24 hours PPTN, ${ }^{*} P=0.029$; IRI 48 hours DMSO vs. IRI 48 hours PPTN, ${ }^{*} P=0.016$; by 2-way ANOVA followed by Tukey's post hoc test. Between 940 and 1700 tubules were analyzed in each group.

solic enzyme carbonic anhydrase type 2 (Car2); V-ATPase subunits including Atp6v1e1, Atp6v1g3, Atp6v1b1, Atp6vod2, Atp6v1f, and Atp6v1h; the solute transporter Slc26a4; and the purinergic receptor P2ry14. qPCR and RNA-Seq showed that following IRI, medullary ICs rapidly adopted a proinflammatory profile characterized by upregulation of inflammatory mediators, including chemokines and cytokines. Whether cortical ICs also have a similar proinflammatory function will require additional studies. These results are in agreement with a previous study showing elevation of cytokines and chemokines in cells isolated by laser capture microdissection from different kidney regions (60). Interestingly, we found that the P2ry14 receptor itself was upregulated after IRI, and we set out to investigate the role of the UDP-Glc/ P2Y14 receptor signaling pathway in the onset of AKI following 
A
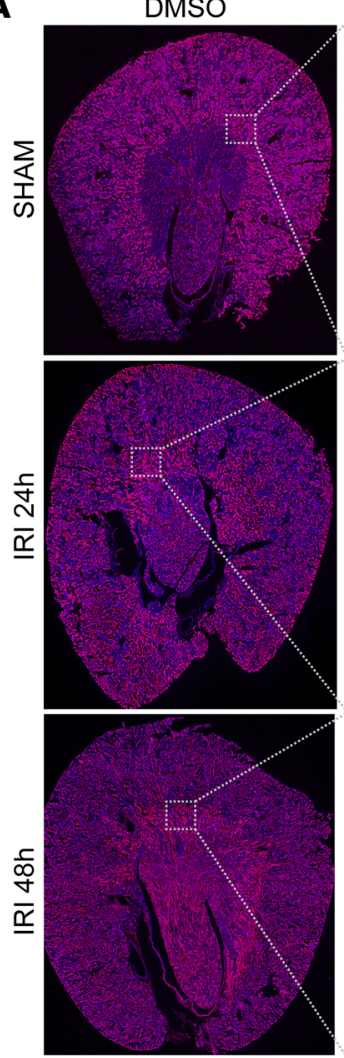

B

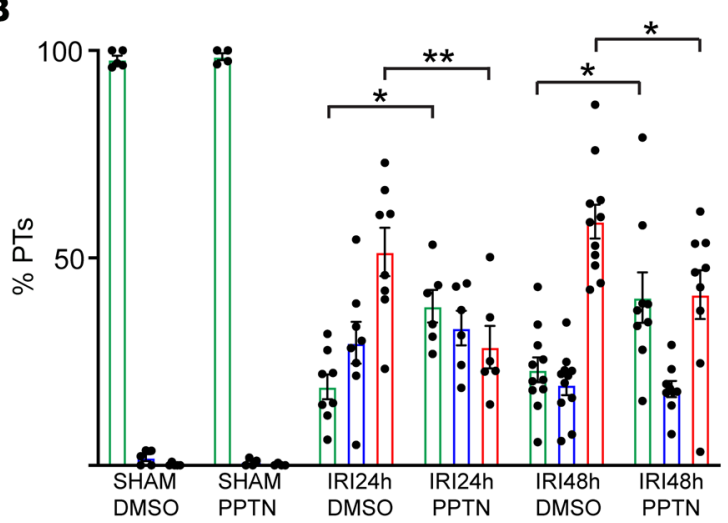

DMSO
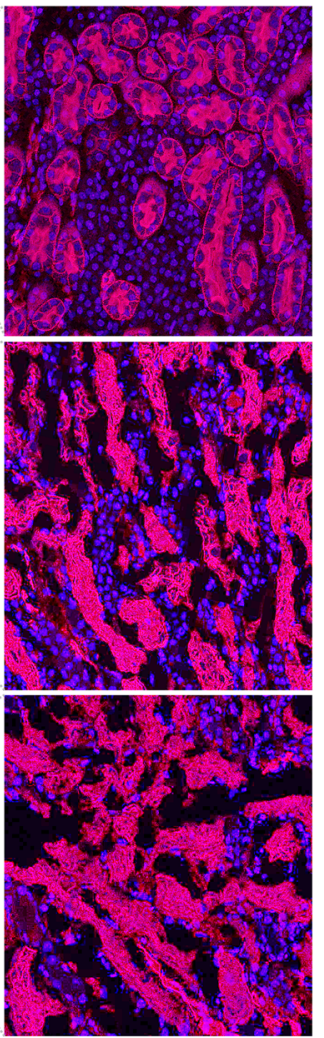

DMSO PPTN
PPTN
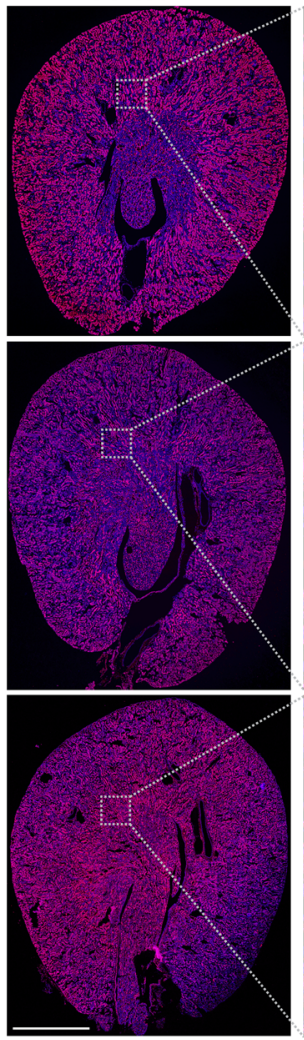

PPTN
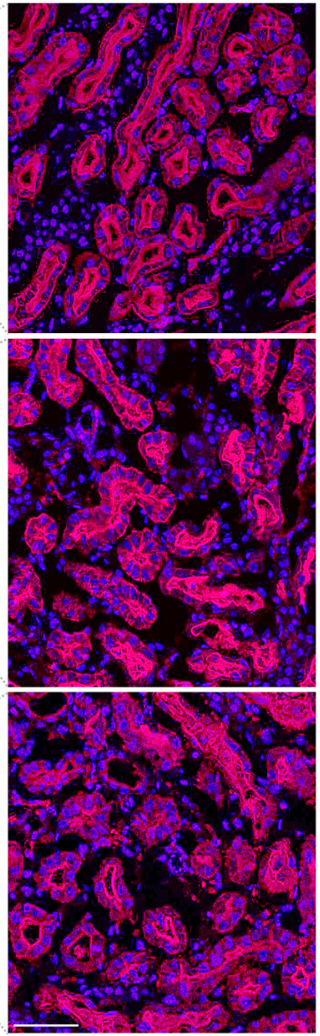

$\square$ Intact PTs

Apical and basolateral AQP1

Moderately damaged PTs

Basolateral AQP1

$\square$ Very damaged PTs

Loss of AQP1 membrane staining
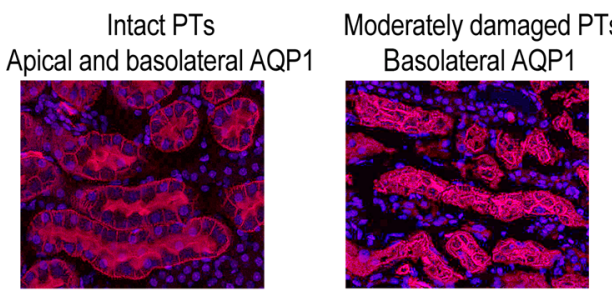

Very damaged PTs Loss of AQP1 membrane staining

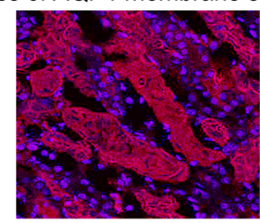

Figure 7. PPTN maintains PT polarity after IRI. (A) Kidney sections labeled for AQP1 showed apical and basolateral localization in PTs from sham-operated mice. Twenty-four hours and 48 hours after IRI, a significant loss of AQP1 polarity was detected. In mice treated with PPTN, several PTs showed intact AQP1 localization at the brush border and basolateral membrane. PPTN alone did not affect AQP1 distribution in sham-operated mice. Scale bar: 1 mm; inset scale bar: $100 \mu \mathrm{m}$. (B) Quantification of the number of intact PTs with apical and basolateral AQP1 labeling (green bars), moderately damaged PTs with loss of apical labeling but detectable basolateral labeling (blue bars), and very damaged PTs with a complete loss of AQP1 polarity (red bars). PPTN induced a significant reduction in the number of very damaged PTs (IRI 24 hours DMSO [ $n=8]$ vs. IRI 24 hours PPTN [ $n=6]{ }^{* *} P=0.0043$, IRI 48 hours DMSO [ $\left.n=11\right]$ vs. IRI 48 hours PPTN $[n=9]{ }^{*} P=0.011$ ) together with an increase in the number of intact PTs 24 hours and 48 hours after IRI (IRI 24 hours DMSO vs. IRI 24 hours PPTN ${ }^{*} P=0.026$, IRI 48 hours DMSO vs. IRI 48 hours PPTN ${ }^{*} P=0.012$, compared with the untreated group; SHAM-DMSO [ $\left.n=5\right]$, SHM-PPTN [ $\left.n=4\right]$ ]). Two-way ANOVA followed by Tukey's post hoc test was performed. Between 1500 and 3000 PTs were analyzed in each group.

renal IRI. We found that UDP-Glc is rapidly excreted by the kidney following IRI, where it is positioned to activate apical P2Y14 receptors. Importantly, PPTN is a small molecule that is excreted intact into the urine, and so it is a suitable therapeutic compound for the prevention/alleviation of AKI. As described below, PPTN has a protective effect not only in the kidney, but also in the lung and uterus, indicating its multiple therapeutic potential.
We show here that renal infiltration of neutrophils and monocytes occurs rapidly, within 2 hours after IRI, in agreement with previous reports (27). Blocking this early response with PPTN protects kidney function and reduces tubular damage, further supporting the notion that inflammation is involved in the pathogenesis of AKI (14). The role of the P2Y14 receptor in triggering renal inflammation following renal IRI is in agreement with our previous 
study showing that a single i.v. injection of UDP-Glc is sufficient to cause renal neutrophil infiltration in healthy mice (42). Other groups have also revealed the proinflammatory role of the P2Y14 receptor in different tissues, including the lung and uterus (41, 43). Patients with cystic fibrosis have elevated levels of UDP-Glc in their lung secretions, and instillation of UDP-Glc into the trachea of WT mice induced lung neutrophil recruitment, a process that was inhibited by PPTN (43). The P2Y14 receptor is expressed in the uterus epithelium, but not in the stroma, in humans and mice, and patients with pelvic inflammatory disease have elevated levels of P2Y14 receptor, IL-8, and IL-1 $\beta$ in their endometrial epithelial lining (41). The renal IRI-induced increase in P2Y14 receptor expression, together with elevated levels of CXCL1 and CXCL2 (the IL-8 murine homologs), IL-1 $\beta$, and other proinflammatory mediators, and the attenuated kidney damage in response to IRI that we observed after silencing the P2Y14 receptor in ICs are in agreement with this previous study, which also showed that knocking down the P2Y14 receptor using siRNAs reduced the number of inflammatory neutrophils in the uterus (41). Collectively, these results indicate that UDP-Glc/P2Y14 receptor signaling occurs across species and that it acts at the luminal surface of organs that are in contact with the external environment. The high stability of UDP-Glc in the extracellular compartment, in contrast to other DAMPs, provides a mechanism by which high extracellular UDP-Glc levels could be achieved following stress and injury (35). This is supported by the rapid elevation of urinary UDP-Glc concentration that we detected after IRI.

The role of epithelial cells in mediating injury is rapidly emerging $(17,27)$. A previous study showed that $\alpha$-ICs protect against urinary pathogenic bacteria by producing the bacteriostatic protein lipocalin 2 (also known as NGAL) (61). Our study provides additional evidence that ICs play a defense role in addition to their traditional role in the regulation of acid/base balance. A previous study showed that ischemic AKI activates the MAPK pathway (60). Interestingly, activation of P2Y14 receptor by UDP-Glc also activates this pathway, further supporting its role in the initiation of AKI $(35,42)$. One of the transcripts that were upregulated in ICs after IRI is IL-1 $\beta$. This cytokine was recently implicated in the initiation and progression of tubulointerstitial fibrosis (62), and it is, therefore, possible that the increase in IL-1 $\beta$ that we show here might contribute to the progression of early inflammation toward fibrosis and might participate in the transition from AKI to chronic kidney disease (CKD). We also detected strong upregulation of IL-34 in ICs, a cytokine that was recently implicated in the onset of AKI and worsening of subsequent CKD (63). While this article described the upregulation of IL-34 by renal tubular cells 3 days after IRI, the present study now shows that this cytokine transcript is rapidly upregulated in ICs and could participate in the very early response to AKI.

Other DAMPs produced in different clinical settings may also induce local organ inflammation (64). For example, ATP, ADP, and UDP are known DAMPS, and they may contribute to the pathophysiology associated with ischemic AKI. Their contribution is tightly regulated by extracellular ectonucleotidases, which metabolize ATP and ADP into adenosine (65). In addition, some pattern recognition receptors are located on the apical surface of tubular cells (e.g., Toll-like receptors [TLRs], P2Y14 recep- tor, etc.), and larger circulatory DAMPS such as the TLR ligand HMGB1 may not cross the glomerular barrier to reach their target. The role of TLRs in AKI has been investigated by several groups. TLR2 activation was proposed to promote PT repair following cisplatin-induced AKI $(66,67)$. Inhibition of TLR2 reduces myocardial IRI in pigs (68). In the kidney, while TLR2 and TLR4 expression is triggered by renal IRI, this increase is preceded by an abrogation of TLR expression 1 and 6 hours after reperfusion $(38,69)$. This indicates that they are probably not involved in the early phase of neutrophil and monocyte extravasation, which occurs within 2 hours after IRI (this study and ref. 27). By contrast, $\mathrm{P} 2 \mathrm{Y} 14$ receptor expression is high at baseline and increases further following IRI, and its ligand UDP-Glc is rapidly excreted by the kidney, indicating its readiness in initiating the very first steps in the inflammatory cascade that accompanies AKI.

Bilateral renal IRI mimics the reduction in renal blood flow that occurs in different hospital settings such as during a direct surgery to remove a kidney tumor, or during transportation of transplanted kidneys. It also models the significant reduction in renal oxygenation that occurs during a cardiac surgery requiring cardiopulmonary bypass (CPB). Indeed, it has been suggested that renal hypoxia secondary to a significant reduction in renal blood flow during $\mathrm{CPB}$ time plays a causative role in post-cardiac surgery AKI (CS-AKI) $(12,21)$. Moreover, heart ischemia occurs in addition to renal ischemia during a cardiac surgery requiring $\mathrm{CPB}$, which would further contribute to the production of DAMPs. Thus, not only a local insult to the kidney, but damage to a remote organ such as the heart during surgery might play a role in CS-AKI. This is supported by the strong correlation that we observed between UDP-Glc and AKI in cardiac surgery patients, in whom both renal and heart ischemia may contribute to AKI via activation of the UDP-Glc/P2Y14 receptor signaling pathway.

Paradoxically, PTs do not express the P2Y14 receptor, but inhibition of this receptor in ICs protects them. The unique architecture of the kidney with collecting ducts, blood vessels, and PTs all running in parallel with each other in the kidney medulla provides a unique morphological environment that is conducive to a cellcell crosstalk between ICs and PTs. It is conceivable that chemokine production specifically in ICs, followed by massive immune cell infiltration, would affect neighboring PT cells, and thus inhibition of this process would reduce the impact of ischemia. Indeed, PTs have the ability to regenerate following ischemia (17), and they would do so more easily in the absence of harmful inflammatory cells. In support of this hypothesis, we observed similar increases in sCr, BUN, and mALB/Cre 2 hours after IRI in both the DMSO and PPTN groups. While sCr remained stable in the PPTN group, it continued to increase after 24 hours in the nontreated group. In addition, similar levels of moderate tubular damage were observed 2 hours after IRI in the DMSO and PPTN groups, but the percentage of very damaged renal tubules (assessed in H\&E sections) and PTs (assessed in AQP1-labeled sections) decreased in the PPTN group compared with DMSO 24 hours after IRI. This was accompanied by a significant increase in the percentage of intact tubules at 48 hours in the PPTN versus DMSO groups. In addition, the reduction in albuminuria and urinary KIM-1 levels that we observed at 48 hours after IRI in the treated group compared with nontreated mice indicated that PPTN attenuated the PT damage caused by IRI. 
A Gated on single live cells

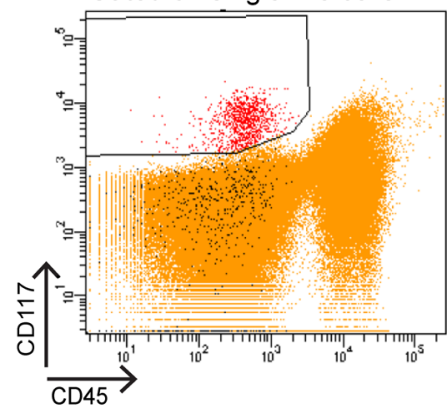

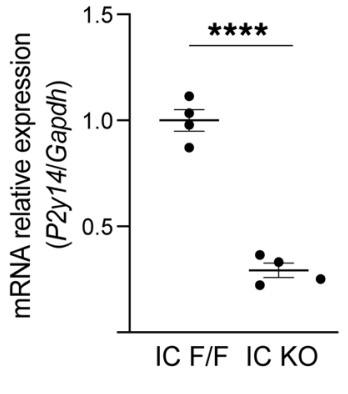

B

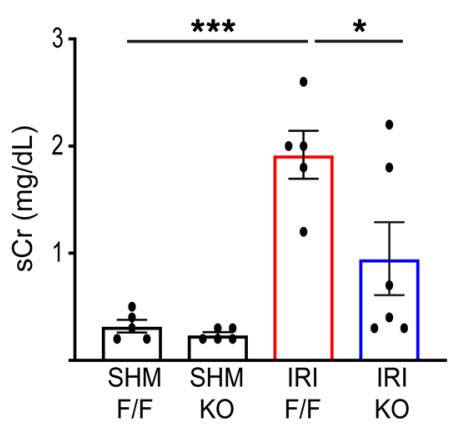

C
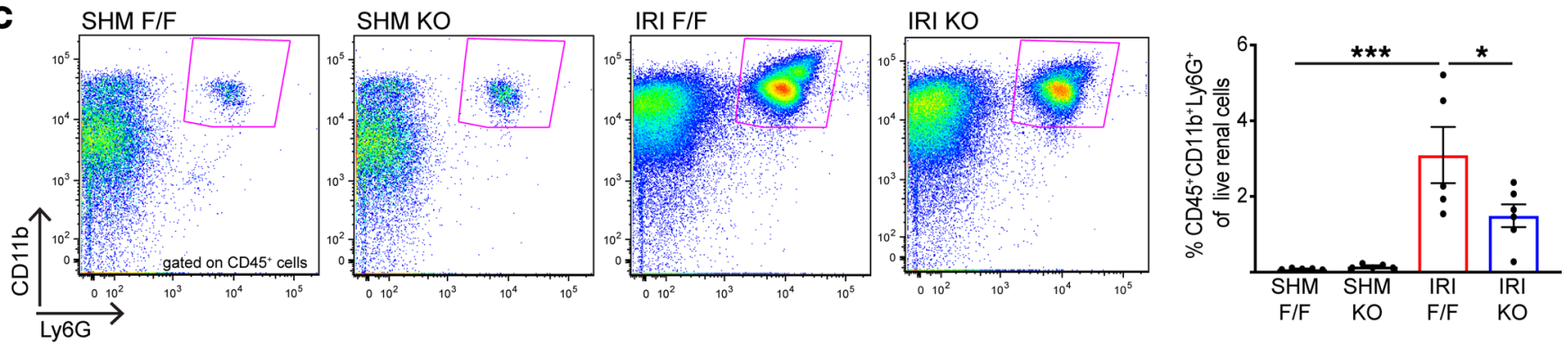

D

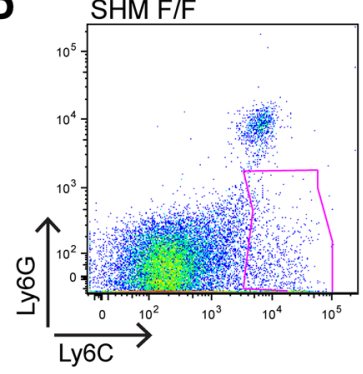

E

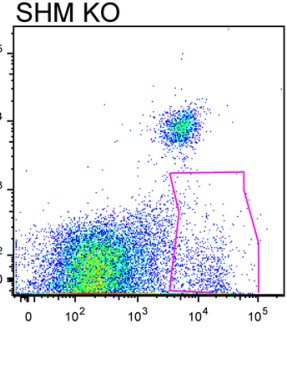

$H \& E$
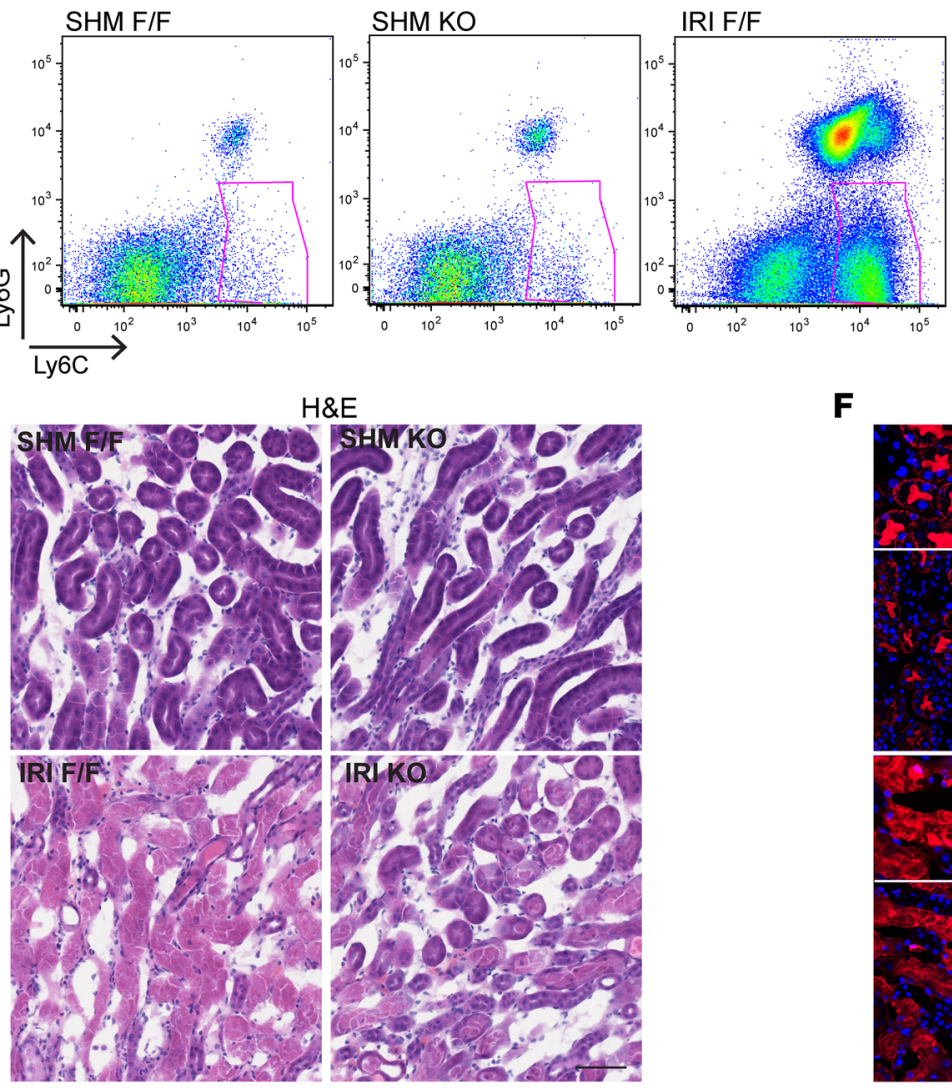

IRI KO
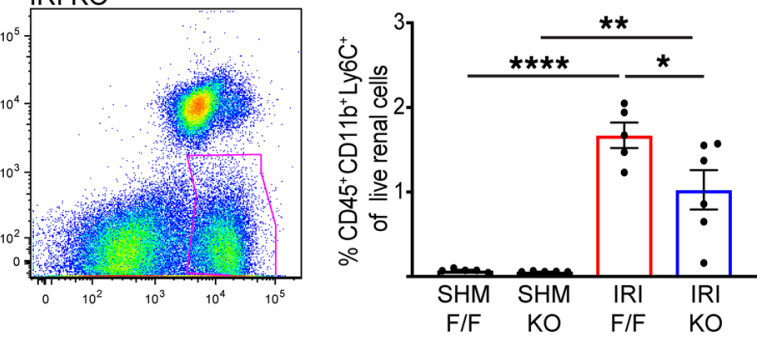

AQP1
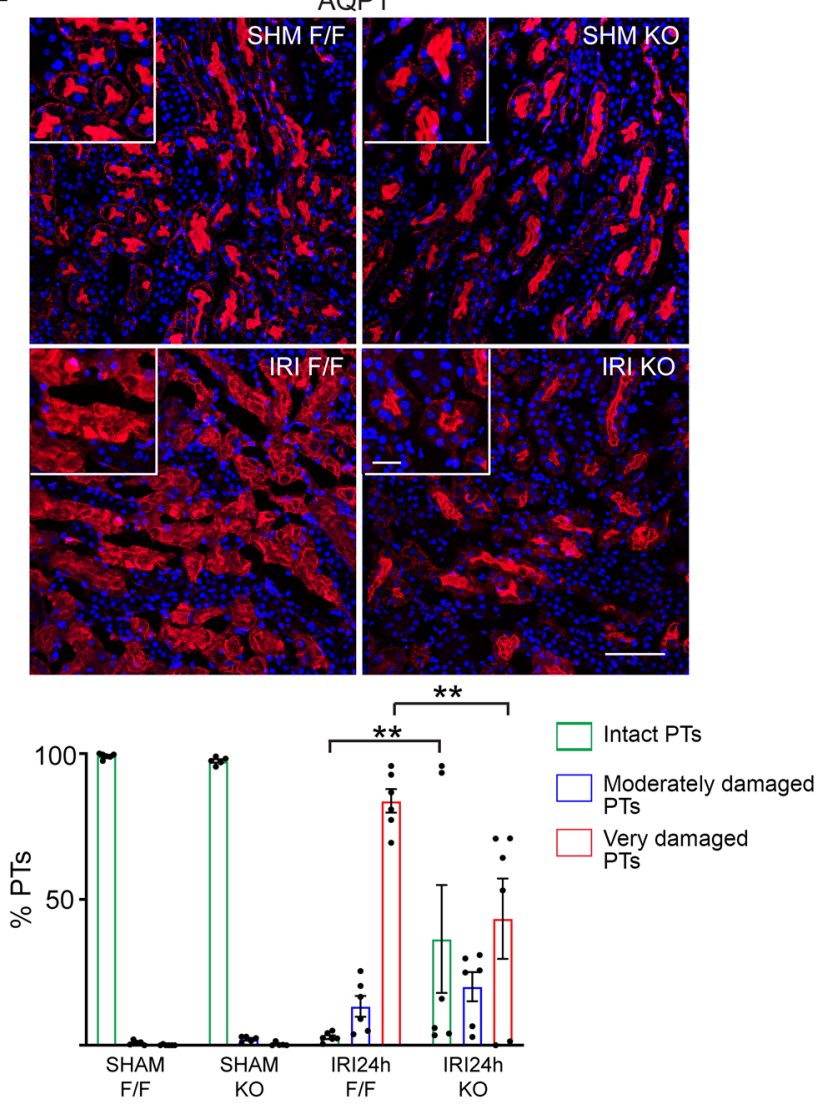
Figure 8. Deletion of P2Y14 in ICs protects kidney function, reduces inflammation, and attenuates damage after IRI. (A) Left: ICs (CD117+ CD45red dots) were isolated from $\mathrm{B}^{\mathrm{Cre}+} \mathrm{P} 2 \mathrm{Y} 14^{\mathrm{fl} /+}$ (IC KO) and $\mathrm{B} 1^{\mathrm{Cre}-} \mathrm{P} 2 \mathrm{Y} 14^{\mathrm{fl} /+}$ (IC F/F; controls) mice. Right: P2ry14 expression by qPCR in IC KO versus IC F/F mice. ${ }^{* * *} P<0.0001$ by unpaired 2-tailed Student's $t$ test $(n=4)$. (B) $s C r$ 24 hours after IRI versus SHAM in F/F and IC KO mice. ${ }^{*} P=0.031$, ${ }^{* *} P=$ 0.0008 . (C) Recruitment of CD45+CD11b+Ly6C $\mathrm{C}^{+}$live cells 24 hours after IRI in $\mathrm{F} / \mathrm{F}$ mice versus SHM $\left({ }^{* *} P=0.0003\right)$, and attenuation in IC KO mice (IRI KO vs. IRI F/F; ${ }^{*} P=0.041$ ). (D) Recruitment of CD45+CD11b+Ly6C+Ly6C- live cells 24 hours after IRI in F/F versus SHM $\left({ }^{* * * *} P<0.0001\right)$, and attenuation in IC KO mice (IRI KO vs. IRI F/F; ${ }^{*} P=0.032$ ). ${ }^{* *} P=0.0014$. (E) H\&E staining of kidney of SHM and 24 hours after IRI in F/F and IC KO mice. Bar graph shows reduction of very damaged tubules (red bars; ${ }^{* *} P=0.0002$ ) and increase in intact tubules (green bars; ${ }^{* *} P=0.006$ ) 24 hours after IRI in KO versus F/F mice. Scale bar: $50 \mu \mathrm{m}$. (F) AQP1 staining of kidney of SHAM and 24 hours after IRI in F/F and IC KO mice. Bar graph shows reduction of very damaged PTs (red bars; ${ }^{* *} P=0.0012$ ) and increase in intact PTs (green bars; ${ }^{* *} P=0.0093$ ) 24 hours after IRI in IC KO versus F/F. Scale bar: $50 \mu \mathrm{m}$; inset: $10 \mu \mathrm{m}$. Data are means \pm SEM. Each dot represents 1 mouse. (B-D) One-way ANOVA followed by Tukey's test. (E and F) Twoway ANOVA followed by Tukey's test. (B-D) $n=5$ for SHM F/F, SHM KO, and IRI KO; $n=6$ for IRI F/F. (E) $n=6$ for IRI F/F, SHM KO, and IRI KO; $n$ $=7$ for SHM F/F. (F) $n=6$ mice for SHAM F/F, IRI 24 hours F/F, and IRI 24 hours KO; $n=5$ for SHAM KO. Between 1400 and 2500 tubules were analyzed per group (E and $\mathbf{F}$ ).

Indeed, both an increased expression of KIM-1 and albuminuria have been previously attributed to PT injury $(53,54)$. Importantly, 24 hours after IRI, many neutrophils were found in close proximity to damaged PTs in the nontreated group, but very few were detected in the PPTN group. Altogether, these results indicate that PPTN confers renal tubule protection following IRI by allowing repair mechanisms to occur and/or by attenuating further damage via reduction of renal inflammation. The latter mechanism is supported by the reduced numbers of apoptotic cells that we detected 24 hours after IRI in the PPTN group versus DMSO.

AKI is a multifactorial disease, and targeted, patient-centered approaches are now proposed to develop effective therapeutics. In this context, enrichment strategies based on biomarkers that are linked to the therapy being tested would allow intervention to a specific group of patients. This would yield a much larger effect size, a true benefit to therapeutics development programs. The fact that UDP-Glc is not only an indicator of AKI, but is also a causal marker that induces early renal inflammation, would provide the advantage of selecting patients who are likely to benefit from the use of a specific inhibitor that would block the UDP-Glc/ P2Y14 receptor proinflammatory pathway. While the IC-specific baseline expression level of the P2Y14 receptor is high (this study and ref. 59), its increased expression following IRI makes it a likely player in the very early steps of inflammation. Importantly, ICs do not appear to be affected by IRI, as they continue to show apical expression of the V-ATPase, in agreement with previous studies showing that collecting ducts are more resistant to ischemia compared with PTs (70). While the current work supports the use of a P2Y14 receptor antagonist to prevent/attenuate AKI following a planned ischemic episode, additional studies will be required to determine whether this strategy could also be used in other clinical settings.

In conclusion, our study provides evidence that inhibiting the UDP-Glc/P2Y14 receptor pathway in ICs plays a protective role in kidneys submitted to an ischemic insult by targeting the early inflammation pathway, a prominent feature of AKI. Our observation that elevated urine levels of UDP-Glc are associated with AKI in ICU and cardiac surgery patients supports further investigation in humans to test the potential therapeutic benefits of inhibiting this pathway to prevent/alleviate AKI in hospital settings.

\section{Methods}

Animals. Adult C57BL/CBAF1 WT male mice were purchased from The Jackson Laboratory. Transgenic mice that express EGFP under the control of the promoter of the IC-specific V-ATPase B1 subunit (ATP6V1B1) gene (71) were also used; they are referred to as B1EGFP mice. Transgenic mice expressing Cre under the control of the ATP6V1B1 promoter $\left(\mathrm{B}^{\mathrm{Cre}}\right)(57,58)$ were bred with P2ry14 Lox mice (B6.129-P2ry14<tm1Gac >/Orl; EMMA, EM:05368). B1 ${ }^{\mathrm{Cre}+}$ P2ry14 $4^{\mathrm{fl} /+}$ (IC KO) and B1 ${ }^{\text {Cre- }}$ P2ry14 ${ }^{\mathrm{fl} /+}$ (IC F/F; controls) mice were generated.

Animal model of IRI. Mice were housed in groups of 3 in metabolic cages (Tecniplast), and were acclimated to the metabolic cages for 4 days before the beginning of the experiment. They received

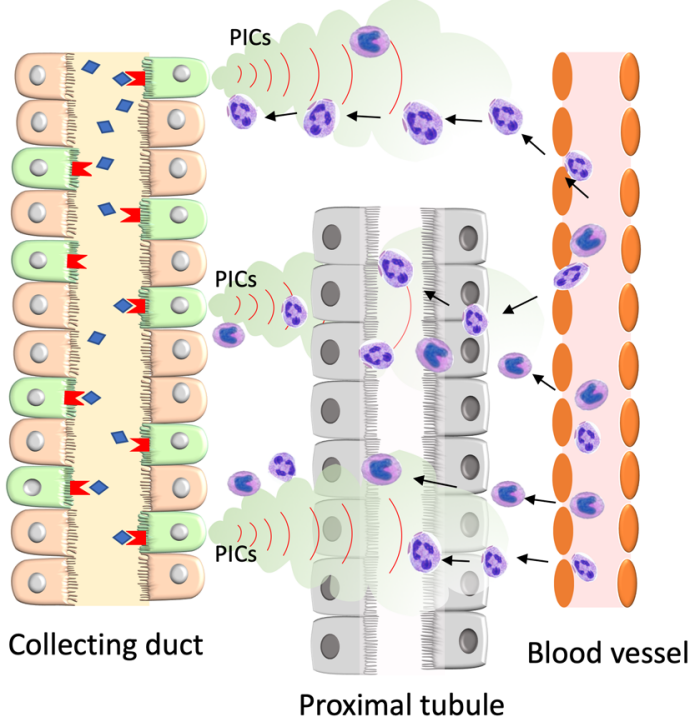

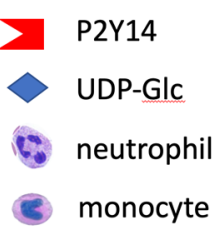

IC

PICs: proinflammatory chemokines
Figure 9. Activation of P2Y14 in ICs triggers renal inflammation leading to PT injury. Renal ischemia induces the release of UDP-Glc from injured cells. UDP-Glc reaches the collecting duct lumen, where it binds P2Y14 located on the apical membrane of ICs. ICS then produce proinflammatory chemokines (PICs), which attract circulating neutrophils and monocytes from the blood vessel into the kidney stroma. Neutrophils and monocytes clog the microvasculature, and extravasated cells attack PT cells, creating additional injury. 


\section{Table 1. Patient characteristics}

$\begin{array}{lcccc} & \text { All patients } & \text { AKI } & \text { No AKI } & P \text { value } \\ & \boldsymbol{n}=\mathbf{3 5} & \boldsymbol{n} \boldsymbol{n = 1 2} & \boldsymbol{n}=\mathbf{2 3} & \\ \text { Age, years } & 73(63,82) & 78(71,86) & 68(63,82) & 0.08 \\ \text { Female (\%) } & 9(26 \%) & 1(8 \%) & 8(35 \%) & 0.22 \\ \text { White (\%) } & 34(97 \%) & 12(100 \%) & 22(96 \%) & 1.00 \\ \text { Reason for ICU admission (\%) } & & & & 0.04 \\ \quad \text { Cardiac surgery } & 26(74 \%) & 6(50 \%) & 20(87 \%) & \\ \quad \text { Sepsis } & 5(14 \%) & 3(25 \%) & 2(9 \%) & \\ \quad \text { Other } & 4(11 \%) & 3(25 \%) & 1(4 \%) & \\ \text { Baseline creatinine, } \mathrm{mg} / \mathrm{dL} & 1(0.9,1.3) & 1.2(1.0,1.6) & 0.9(0.8,1.1) & 0.04 \\ \text { Peak creatinine, } \mathrm{mg} / \mathrm{dL} & 1.1(0.9,1.5) & 1.8(1.4,2.8) & 0.9(0.8,1.1) & <0.001\end{array}$

Continuous variables are presented as median (IQR).

4-[4-(4-piperidinyl)phenyl]-7-[4-(trifluoromethyl)phenyl]-2-naphthalenecarboxylic acid hydrochloride (PPTN-HCl; Tocris, cata$\log 4862$ ) or vehicle (DMSO). Mice were divided into 4 groups (SHAM-DMSO, SHAM-PPTN, IRI-DMSO, and IRI-PPTN). For the 2-hour time point, mice were treated via a single injection through the tail vein, and for the 24-hour and 48-hour time points, they were treated via osmotic minipumps (ALZET) implanted subcutaneously 1 day before the day of IRI surgery.

On the day of bilateral IRI surgery, mice were anesthetized with isoflurane and the kidneys were exposed through flank incisions. The renal pedicle was clamped with an atraumatic vascular clip during 35 minutes. After the IRI surgery, animals were placed back into metabolic cages and allowed to recover for 2 hours, 4 hours, 24 hours, or 48 hours.
At the end of the experimental period, mice were anesthetized with pentobarbital (60 $\mathrm{mg} / \mathrm{kg}$ body, i.p.), and a blood sample was collected from the left cardiac ventricle. Mice were perfused via the left cardiac ventricle with PBS until kidneys were cleared of blood. The left kidney artery was clamped, and the left kidney was harvested and processed for flow cytometry analysis. The perfusion continued with paraformaldehyde-lysineperiodate fixative (PLP) as previously described (72). The right kidney was used for histopathology and immunofluorescence analysis.

UDP-Glc measurement by LC-MS/MS. UDP-Glc concentration was measured in mouse urine samples using the LC-MS/MS method performed at the Small Molecule Mass Spectrometry Facility, Faculty of Arts and Sciences, Harvard University (Cambridge, Massachusetts, USA). The parameters used are listed in Supplemental Methods. Isolation of EGFP $P^{+}$ICs from B1-EGFP mice, RNA extraction, and RNA-Seq. Isolation of $\mathrm{EGFP}^{+} \mathrm{ICs}$ from the renal medulla of B1-EGFP mice was performed as we previously described (72). The RNA of EGFP $^{+}$ICs from SHAM or IRI animals was isolated using a PicoRNA Kit (Thermo Fisher Scientific). Each sample was obtained using 2 kidneys (from 2 mice). RNA-Seq libraries were prepared using the Clontech SMARTER Kit v4, followed by sequencing on an Illumina HiSeq2500 instrument. Transcriptome mapping was performed with STAR (73) using the Ensembl annotation of $\mathrm{mm} 9$ reference genome. Read counts for individual genes were produced using HTSeq (74). Differential expression analysis was performed using the EdgeR package (75) after normalization of read counts and inclusion of only those genes with counts per million greater than 1 for one or more samples.
Figure 10. Elevated UDP-GIc urinary levels are associated with AKI in ICU and cardiac surgery patients. (A) Average peak urinary UDP-Glc concentration in ICU patients with AKI (ICU-AKI, $n=12$ ) and without AKI (No AKI, $n=23$ ). Significantly higher UDP-GIc concentration was detected in patients who developed AKI versus patients who did not. (B) Average peak urinary UDP-GIc concentration in cardiac surgery patients with AKI (CS-AKI, $n=6$ ) and without AKI (No AKI, $n=20$ ). Significantly higher UDP-Glc concentration was detected in patients who developed AKI versus patients who did not. (c) Receiver operating characteristic (ROC) curve for the diagnosis of AKI (stages 1, 2, and 3 combined) in ICU patients. Peak UDP-GIc levels versus the higher AKI stage for each patient were compared. (D) ROC curve for the diagnosis of AKI (stages 1, 2, and 3 combined) in cardiac surgery patients. Data are expressed as median \pm IQR for continuous variables from a cohort of 35 patients. (A and B) Statistical analysis was performed using Wilcoxon's rank-sum test, $\chi^{2}$ test, and Fisher exact test. (C and D) A $\chi^{2}$ test was performed to compare the area under the ROC curve (AUC) with that of an intercept-only model, which has an AUC of 0.5 . Two-sided $P$ values less than 0.05 were considered statistically significant.
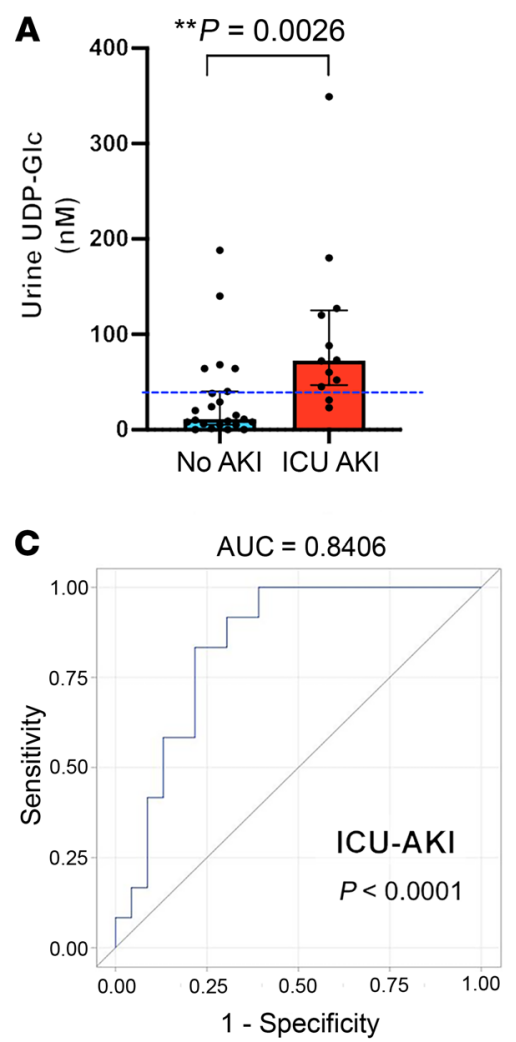
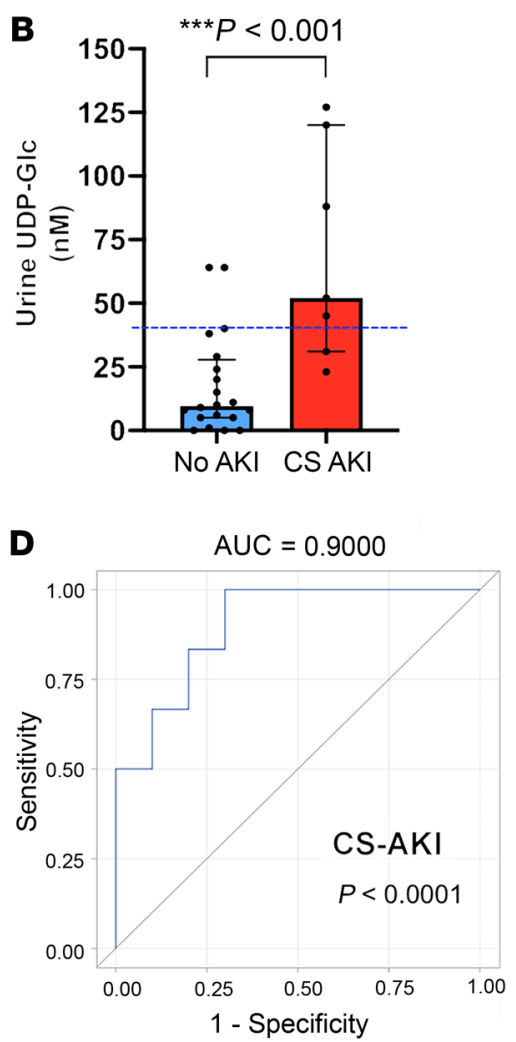
Differentially expressed genes were defined based on the criteria of more than 2 -fold change in expression value, $P$ less than 0.05 , and coefficient of variation less than 1.2. Multiplot studio software was used to obtain differential gene expression. RNA-Seq data sets from SHAM and IRI ICs were deposited in the NCBI's Gene Expression Omnibus database (GEO GS144522).

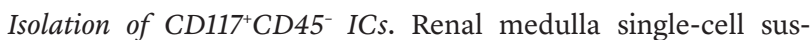
pensions were generated from $\mathrm{B} 1^{\mathrm{Cre}+} P 2 r y 14^{\mathrm{I} /+}$ mice (IC KO) and $\mathrm{B}^{\mathrm{Cre}-} \mathrm{P} 2 \mathrm{ry} 14^{\mathrm{fl} /+}$ mice (IC F/F; controls), as described in Supplemental Methods for the $\mathrm{EGFP}^{+} \mathrm{IC}$ isolation procedure. Cell suspensions were incubated with anti-mouse antibodies (1:100) against PE/Cy7-CD117 (clone 2B8) and BV711-CD45 (clone 30-F11). Antibodies were purchased from BD Biosciences, FACS isolation of CD $117^{+} \mathrm{CD} 45^{-} \mathrm{ICs}$ from the renal medulla was performed at the Harvard Stem Cell InstituteCenter for Regenerative Medicine Flow Cytometry Core. The RNA of $\mathrm{CD} 177^{+} \mathrm{CD} 45^{-}$ICs was isolated using a PicoRNA Kit (Thermo Fisher Scientific). Each sample was obtained using 2 kidneys (1 mouse).

Proinflammatory molecule expression in EGFP ${ }^{+}$ICs by qPCR. EGFP ${ }^{+}$ ICs were isolated by FACS from B1-EGFP mice 2 hours, 4 hours, and 24 hours after IRI or sham surgery, and total RNA was isolated from EGFP cells as described in Supplemental Methods. cDNA was synthesized from 1000 pg RNA using the SuperScript VILO cDNA Synthesis Kit (Invitrogen) according to the manufacturer's instructions. Quantitative real-time PCR was performed using the Power SYBR Green PCR Master Mix (Life Technologies) and primers listed in Supplemental Table 4. Results are reported as mean \pm SEM using the formula $-\Delta \mathrm{Ct}=-[\mathrm{Ct}$ target gene - Ct control gene Gapdh]. Relative expression is derived from $2^{-\Delta \Delta C t}$, where $-\Delta \Delta \mathrm{Ct}=\Delta \mathrm{Ct}$ treated group - mean of $\Delta \mathrm{Ct}$ control group.

PPTN measurement. The urine concentration of PPTN was quantified by LC-MS/MS at the Small Molecule Mass Spectrometry Facility, Faculty of Arts and Sciences, Harvard University, as described in detail in Supplemental Methods.

Flow cytometry analysis. Renal medulla single-cell suspensions were generated as described previously (72) and in Supplemental Methods. Cell suspensions were incubated with anti-mouse antibodies (1:100) against PE/Cy7-F4/80 (clone BM8), BV711-CD45 (clone 30-F11), APC/Cy7-CD11b (clone M1/711), FITC-LY6C (clone AL-21), PE-LY6G (clone 1A8), and Alexa Fluor 647-CD64 (clone X54-5/7.1). Antibodies were purchased from BD Biosciences or BioLegend.

Measurement of urinary and serum markers of kidney function. Whole-blood samples were allowed to clot 30 minutes at room temperature before centrifugation at $4000 \mathrm{~g}$ for 10 minutes for collection of serum. Blood urea nitrogen (BUN) and serum creatinine ( $\mathrm{sCr}$ ) were measured using a Heska DriChem 7000 chemical analyzer (http:// www.heska.com/product/element-dc5x), and urine creatinine and microalbuminuria were measured using the DCA Vantage Analyzer (Siemens Healthineers), available at the Center for Comparative Medicine of Massachusetts General Hospital. Urinary KIM-1 concentration was measured using the mouse KIM-1 ELISA (R\&D Systems, catalog MKM100).

Immunocytochemistry (H\&E) and immunofluorescence. PLP-fixed kidney slices were processed for cryosectioning, immunocytochemistry, and immunofluorescence. Kidney sections were labeled using $\mathrm{H} \& \mathrm{E}$, phalloidin (marker of F-actin), and antibodies against AQP1 and megalin (PT markers), V-ATPase B1 subunit (IC marker), N-elastase and Ly6G (neutrophil markers), and cleaved caspase-3 (apoptotic marker), as described in detail in Supplemental Methods.
The percentage of intact, moderately damaged, and very damaged renal tubules was quantified in H\&E-stained sections, and the percentage of intact, moderately damaged, and very damaged PTs was quantified in AQP1-labeled sections by 2 investigators who were blinded to the study. The following AQP1 staining patterns were used: Intact PTs were identified when AQP1 was located in the brush border and basolateral membrane. A complete loss of AQP1 polarity with absent apical and basolateral staining indicated very damaged PTs. A partial redistribution of AQP1 with basolateral staining still visible but loss of apical staining indicated moderately damaged PTs. The number of apoptotic cells was quantified by counting of the number of cells positive for cleaved caspase-3 per area of tissue $\left(16,900 \mu \mathrm{m}^{2}\right)$ in deidentified samples by 2 people.

ICU patient pilot study. This prospective pilot study was conducted at Massachusetts General Hospital. Exclusion criteria were age under 18 years, pregnancy, long-term or acute dialysis, and organ transplantation within the prior year. Written informed consent was obtained from all study participants. Medical records were reviewed prospectively to retrieve baseline demographic characteristics and preoperative clinical and laboratory variables, including serial $\mathrm{sCr}$ measurements. Urine samples were freshly collected daily, and collection started the time of arrival at the ICU for the patients not requiring cardiac surgery, or before surgery for the cardiac surgery group.

Urine samples were centrifuged to remove cells and cell debris, and urinary aliquots were stored at $-80^{\circ} \mathrm{C}$ until assayed. Urinary UDPGlc concentration was measured using the UDP-Glc pyrophosphorylase-catalyzed conversion of UDP-Glc and ${ }^{32} \mathrm{P}$-labeled pyrophosphate $\left.{ }^{(22} \mathrm{PPi}\right)$ to $\left[{ }^{32} \mathrm{P}\right] \mathrm{UTP}$ and glucose-1P, as previously described $(43,76)$, and as detailed in Supplemental Methods.

Statistics. The numeric data were analyzed using GraphPad Prism (version 8; GraphPad Software). Data were analyzed using Student's $t$ test (2 tailed), 1-way ANOVA, or 2-way ANOVA followed by Tukey's or Dunnett's post hoc tests. A $P$ value less than 0.05 was considered significant. Data were expressed as the means \pm SEM. For each set of data, at least 4 different animals were examined for each condition. Collection, analysis, and interpretation of data were conducted by at least 2 independent investigators, who were blinded to the study.

Patient characteristics were described as median $\pm \mathrm{IQR}$ for continuous variables, and counts and percentages for categorical variables. The differences between patients who developed AKI and those who did not were compared using Wilcoxon's rank-sum test, $\chi^{2}$ test, and Fisher exact test, as appropriate. We also drew the ROC curves for all patients, and for cardiac surgery patients (77). A $\chi^{2}$ test was performed to compare the area under the ROC curve (AUC) with that of an intercept-only model, which has an AUC of 0.5. Analyses were conducted using SAS 9.4, and 2 -sided $P$ values less than 0.05 were considered statistically significant.

Study approval. All preclinical procedures were approved by the Massachusetts General Hospital (MGH) Subcommittee on Research Animal Care and were performed in accordance with the NIH Guide for the Care and Use of Laboratory Animals (National Academies Press, 2011; protocol 2015N000016). The patient pilot study was approved by the MGH IRB (protocol 2015P000511).

\section{Author contributions}

MAB, ASA, SK, DB, and SB designed the study. MAB, ACM, RGS, RNL, and JS performed the experiments and analyzed the data. $\mathrm{MAB}, \mathrm{DB}$, and SB wrote the manuscript. MAB, ACM, RGS, ASA, 
SK, SMW, JVB, ERL, DB, and SB read and commented on various drafts of the manuscript. RNL was trained in the laboratory of JVB in the renal IRI procedure.

\section{Acknowledgments}

This work was supported by NIH grants HD040793 and 5U54HL119145 (toSB), R01DK121848 (to DB), DK119793 (toSMW), and P01HL110873 (to ERL). SB is the recipient of the Richard Moerschner Endowed MGH Research Institute Chair in Men's Health and is supported by a grant from Danone Nutricia Research. ASA is supported by American Heart Association award 18CDA34110131. The Microscopy Core facility of the Massachusetts General Hospital (MGH) Program in Membrane Biology receives support from the Boston Area Diabetes and Endocrinology Research Center, grant
DK57521, and the Center for the Study of Inflammatory Bowel Disease, grant DK43351. The Zeiss LSM800 microscope was acquired using an NIH Shared Instrumentation Grant, S10-OD-021577-01. We thank the Harvard Stem Cell Institute-Center for Regenerative Medicine Flow Cytometry Facility (MGH), and in particular Maris Handley and Amy Galvin Watt, for their guidance and assistance in sorting and flow cytometry analysis.

Address correspondence to: Sylvie Breton, Simches Research Center, 185 Cambridge Street Suite 8.204, Boston, Massachusetts 02114, USA. Email: breton.sylvie@mgh.harvard.edu.

JS's present address is: CONICET, Facultad de Ciencias Médicas, Universidad Nacional de Rosario, Rosario, Argentina.
1. Brown JR, Rezaee ME, Marshall EJ, Matheny ME. Hospital mortality in the United States following acute kidney injury. Biomed Res Int . 2016;2016:4278579.

2. Kashani K, et al. Quality improvement goals for acute kidney injury. Clin J Am Soc Nephrol. 2019;14(6):941-953.

3. Hoste EA, et al. Epidemiology of acute kidney injury in critically ill patients: the multinational AKI-EPI study. Intensive Care Med. 2015;41(8):1411-1423.

4. Zuk A, Bonventre JV. Recent advances in acute kidney injury and its consequences and impact on chronic kidney disease. Curr Opin Nephrol Hypertens. 2019;28(4):397-405.

5. Lameire $\mathrm{NH}$, et al. Acute kidney injury: an increasing global concern. Lancet. 2013;382(9887):170-179.

6. Siew ED, Davenport A. The growth of acute kidney injury: a rising tide or just closer attention to detail? Kidney Int. 2015;87(1):46-61.

7. Benoit SW, Devarajan P. Acute kidney injury: emerging pharmacotherapies in current clinical trials. Pediatr Nephrol. 2018;33(5):779-787.

8. Hulse M, Rosner MH. Drugs in development for acute kidney injury. Drugs. 2019;79(8):811-821.

9. Ostermann M, Liu K, Kashani K. Fluid management in acute kidney injury. Chest. 2019;156(3):594-603.

10. Buglioni A, Burnett JC. Pathophysiology and the cardiorenal connection in heart failure. Circulating hormones: biomarkers or mediators. Clin Chim Acta. 2015;443:3-8.

11. Harel Z, Chan CT. Predicting and preventing acute kidney injury after cardiac surgery. Curr Opin Nephrol Hypertens. 2008;17(6):624-628.

12. Lannemyr L, Bragadottir G, Krumbholz V, Redfors B, Sellgren J, Ricksten SE. Effects of cardiopulmonary bypass on renal perfusion, filtration, and oxygenation in patients undergoing cardiac surgery. Anesthesiology. 2017;126(2):205-213.

13. O'Neal JB, Shaw AD, Billings FT. Acute kidney injury following cardiac surgery: current understanding and future directions. Crit Care. 2016;20(1):187.

14. Singbartl K, Formeck CL, Kellum JA. Kidney-immune system crosstalk in AKI. Semin Nephrol. 2019;39(1):96-106.

15. O'Connor ME, Hewson RW, Kirwan CJ, Ackland GL, Pearse RM, Prowle JR. Acute kidney injury and mortality 1 year after major non-cardiac sur- gery. Br J Surg. 2017;104(7):868-876.

16. Wang Y, Bellomo R. Cardiac surgery-associated acute kidney injury: risk factors, pathophysiology and treatment. Nat Rev Nephrol. 2017;13(11):697-711.

17. Bonventre JV, Yang L. Cellular pathophysiology of ischemic acute kidney injury. J Clin Invest. 2011;121(11):4210-4221.

18. Ascione R, Lloyd CT, Underwood MJ, Gomes WJ, Angelini GD. On-pump versus off-pump coronary revascularization: evaluation of renal function. Ann Thorac Surg. 1999;68(2):493-498.

19. Bucerius J, et al. On-pump versus off-pump coronary artery bypass grafting: impact on postoperative renal failure requiring renal replacement therapy. Ann Thorac Surg. 2004;77(4):1250-1256.

20. Nigwekar SU, Kandula P, Hix JK, Thakar CV. Off-pump coronary artery bypass surgery and acute kidney injury: a meta-analysis of randomized and observational studies. Am J Kidney Dis. 2009;54(3):413-423.

21. Ranucci M, et al. Oxygen delivery during cardiopulmonary bypass and acute renal failure after coronary operations. Ann Thorac Surg. 2005;80(6):2213-2220.

22. Awad AS, et al. Compartmentalization of neutrophils in the kidney and lung following acute ischemic kidney injury. Kidney Int. 2009;75(7):689-698.

23. Black LM, Lever JM, Agarwal A. Renal inflammation and fibrosis: a double-edged sword. J Histochem Cytochem. 2019;67(9):663-681.

24. Lee SA, et al. Characterization of kidney CD $45^{\text {int }}{ }^{-} D 11 b^{\text {int }} \mathrm{F} 4 / 80^{+} \mathrm{MHCII}^{+} \mathrm{CX} 3 \mathrm{CR} 1^{+} \mathrm{Ly} 6 \mathrm{C}$ "intermediate mononuclear phagocytic cells". PLoS One. 2018;13(6):e0198608.

25. Lever JM, et al. Resident macrophages reprogram toward a developmental state after acute kidney injury. JCI Insight. 2019;4(2):125503.

26. Williams TM, Wise AF, Layton DS, Ricardo SD. Phenotype and influx kinetics of leukocytes and inflammatory cytokine production in kidney ischemia/reperfusion injury. Nephrology (Carlton). 2018;23(1):75-85.

27. Basile DP, Anderson MD, Sutton TA. Pathophysiology of acute kidney injury. Compr Physiol. 2012;2(2):1303-1353.

28. Imig JD, Ryan MJ. Immune and inflammatory role in renal disease. Compr Physiol.
2013;3(2):957-976.

29. Rabb H, et al. Inflammation in AKI: current understanding, key questions, and knowledge gaps. J Am Soc Nephrol. 2016;27(2):371-379.

30. Bolisetty S, Agarwal A. Neutrophils in acute kidney injury: not neutral any more. Kidney Int. 2009;75(7):674-676.

31. Bonventre JV, et al. AKI: a path forward. Clin JAm Soc Nephrol. 2013;8(9):1606-1608.

32. Chen GY, Nuñez G. Sterile inflammation: sensing and reacting to damage. Nat Rev Immunol. 2010;10(12):826-837.

33. Kono H, Rock KL. How dying cells alert the immune system to danger. Nat Rev Immunol. 2008;8(4):279-289.

34. Schaefer L. Complexity of danger: the diverse nature of damage-associated molecular patterns. J Biol Chem. 2014;289(51):35237-35245.

35. Lazarowski ER, Harden TK. UDP-sugars as extracellular signaling molecules: cellular and physiologic consequences of $\mathrm{P} 2 \mathrm{Y} 14$ receptor activation. Mol Pharmacol. 2015;88(1):151-160.

36. Lazarowski ER, Sesma JI, Seminario L, Esther CR, Kreda SM. Nucleotide release by airway epithelia. Subcell Biochem. 2011;55:1-15.

37. Lazarowski ER, Shea DA, Boucher RC, Harden TK. Release of cellular UDP-glucose as a potential extracellular signaling molecule. Mol Pharmacol. 2003;63(5):1190-1197.

38. Wu H, et al. TLR4 activation mediates kidney ischemia/reperfusion injury. J Clin Invest. 2007;117(10):2847-2859.

39. Vallon V, Unwin R, Inscho EW, Leipziger J, Kishore BK. Extracellular nucleotides and $\mathrm{P} 2$ receptors in renal function. Physiol Rev. 2020;100(1):211-269.

40. Sesma JI, et al. Endoplasmic reticulum/golgi nucleotide sugar transporters contribute to the cellular release of UDP-sugar signaling molecules. J Biol Chem. 2009;284(18):12572-12583.

41. Arase T, et al. The UDP-glucose receptor P2RY14 triggers innate mucosal immunity in the female reproductive tract by inducing IL-8. J Immunol. 2009;182(11):7074-7084.

42. Azroyan A, et al. Renal intercalated cells sense and mediate inflammation via the $\mathrm{P} 2 \mathrm{Y} 14$ receptor. PLoS One. 2015;10(3):e0121419.

43. Sesma JI, et al. UDP-glucose promotes neutrophil recruitment in the lung. Purinergic Signal. 
2016;12(4):627-635

44. Sesma JI, et al. The UDP-sugar-sensing P2Y(14) receptor promotes Rho-mediated signaling and chemotaxis in human neutrophils. Am J Physiol Cell Physiol. 2012;303(5):C490-C498.

45. Zimmermann H. Extracellular metabolism of ATP and other nucleotides. Naunyn Schmiedebergs Arch Pharmacol. 2000;362(4-5):299-309.

46. Chambers JK, et al. A G protein-coupled receptor for UDP-glucose. J Biol Chem. 2000;275(15):10767-10771.

47. Breton S, Brown D. Novel proinflammatory function of renal intercalated cells. Ann Nutr Metab. 2018;72(suppl 2):11-16

48. Brown D, Lee R, Bonventre JV. Redistribution of villin to proximal tubule basolateral membranes after ischemia and reperfusion. Am J Physiol. 1997;273(6):F1003-F1012.

49. Barrett MO, et al. A selective high-affinity antagonist of the P2Y14 receptor inhibits UDP-glucose-stimulated chemotaxis of human neutrophils. Mol Pharmacol. 2013;84(1):41-49.

50. Jacobson KA, Müller CE. Medicinal chemistry of adenosine, $\mathrm{P} 2 \mathrm{Y}$ and $\mathrm{P} 2 \mathrm{X}$ receptors. Neuropharmacology. 2016;104:31-49.

51 . Sabbisetti VS, et al. Blood kidney injury molecule-1 is a biomarker of acute and chronic kidney injury and predicts progression to ESRD in type I diabetes. J Am Soc Nephrol. 2014;25(10):2177-2186.

52. Spiegel DM, Wilson PD, Molitoris BA. Epithelial polarity following ischemia: a requirement for normal cell function. Am J Physiol. 1989;256(3 pt 2):F430-F436.

53. Han WK, Bailly V, Abichandani R, Thadhani R, Bonventre JV. Kidney Injury Molecule-1 (KIM-1): a novel biomarker for human renal proximal tubule injury. Kidney Int. 2002;62(1):237-244.

54. Wagner MC, et al. Proximal tubules have the capacity to regulate uptake of albumin. JAm Soc Nephrol. 2016;27(2):482-494.

55 . Schwartz N, et al. Ischemia activates actin depolymerizing factor: role in proximal tubule microvillar actin alterations. Am J Physiol.
1999;276(4):F544-F551.

56. Sabolić I, et al. Localization of the CHIP28 water channel in rat kidney. Am J Physiol. 1992;263(6 pt 1):C1225-C1233.

57. Miller RL, et al. The V-ATPase B1-subunit promoter drives expression of Cre recombinase in intercalated cells of the kidney. Kidney Int 2009;75(4):435-439.

58. Nanami M, et al. The role of intercalated cell Nedd4-2 in BP regulation, ion transport, and transporter expression. J Am Soc Nephrol. 2018;29(6):1706-1719.

59. Chen L, et al. Transcriptomes of major renal collecting duct cell types in mouse identified by single-cell RNA-seq. Proc Natl Acad Sci US A. 2017;114(46):E9989-E9998.

60. Xu K, et al. Unique transcriptional programs identify subtypes of AKI. J Am Soc Nephrol. 2017;28(6):1729-1740.

61. Paragas $\mathrm{N}$, et al. $\alpha$-Intercalated cells defend the urinary system from bacterial infection. J Clin Invest. 2014;124(7):2963-2976.

62. Lemos DR, et al. Interleukin-1 $\beta$ activates a MYC-dependent metabolic switch in kidney stromal cells necessary for progressive tubulointerstitial fibrosis. J Am Soc Nephrol. 2018;29(6):1690-1705.

63. Baek JH, et al. IL-34 mediates acute kidney injury and worsens subsequent chronic kidney disease. J Clin Invest. 2015;125(8):3198-3214.

64. Meissner M, Viehmann SF, Kurts C. DAMPening sterile inflammation of the kidney. Kidney Int. 2019;95(3):489-491.

65. Eltzschig HK, Sitkovsky MV, Robson SC. Purinergic signaling during inflammation. $N$ Engl J Med. 2013;368(13):1260.

66. Sallustio F, et al. Inhibin-A and decorin secreted by human adult renal stem/progenitor cells through the TLR2 engagement induce renal tubular cell regeneration. Sci Rep. 2017;7(1):8225.

67. Andrade-Silva M, et al. TLR2 and TLR4 play opposite role in autophagy associated with cisplatin-induced acute kidney injury. Clin Sci.
2018;132(16):1725-1739.

68. Arslan F, et al. Treatment with OPN-305, a humanized anti-Toll-Like receptor-2 antibody, reduces myocardial ischemia/reperfusion injury in pigs. Circ Cardiovasc Interv. 2012;5(2):279-287.

69. Wolfs TG, et al. In vivo expression of Toll-like receptor 2 and 4 by renal epithelial cells: IFN- $\gamma$ and TNF- $\alpha$ mediated up-regulation during inflammation. JImmunol. 2002;168(3):1286-1293.

70. Witzgall R, Brown D, Schwarz C, Bonventre JV. Localization of proliferating cell nuclear antigen, vimentin, c-Fos, and clusterin in the postischemic kidney. Evidence for a heterogenous genetic response among nephron segments, and a large pool of mitotically active and dedifferentiated cells. J Clin Invest. 1994;93(5):2175-2188.

71. Miller RL, et al. V-ATPase B1-subunit promoter drives expression of EGFP in intercalated cells of kidney, clear cells of epididymis and airway cells of lung in transgenic mice. Am J Physiol Cell Physiol. 2005;288(5):C1134-C1144.

72. Battistone MA, et al. Extracellular adenosine stimulates vacuolar ATPase-dependent proton secretion in medullary intercalated cells. JAm Soc Nephrol. 2018;29(2):545-556.

73. Dobin A, et al. STAR: ultrafast universal RNA-seq aligner. Bioinformatics. 2013;29(1):15-21.

74. Anders S, Pyl PT, Huber W. HTSeq - a Python framework to work with high-throughput sequencing data. Bioinformatics. 2015;31(2):166-169.

75. Robinson MD, McCarthy DJ, Smyth GK. edgeR: a Bioconductor package for differential expression analysis of digital gene expression data. Bioinformatics. 2010;26(1):139-140.

76. Lazarowski ER, Boucher RC, Harden TK. Mechanisms of release of nucleotides and integration of their action as P2X- and P2Y-receptor activating molecules. Mol Pharmacol. 2003;64(4):785-795.

77. DeLong ER, DeLong DM, Clarke-Pearson DL. Comparing the areas under two or more correlated receiver operating characteristic curves: a nonparametric approach. Biometrics. 1988;44(3):837-845. 